Electronic version of an article published as:

Math. Models Methods Appl. Sci. 25, 1011 (2015). DOI: 10.1142/S0218202515500268

(c) World Scientific Publishing Company, http://www.worldscientific.com/worldscinet/m3as

\title{
FORMAL ASYMPTOTIC LIMIT OF A DIFFUSE-INTERFACE TUMOR-GROWTH MODEL
}

\author{
DANIELLE HILHORST \\ CNRS, Laboratoire de Mathématique, Université de Paris-Sud \\ F-91405 Orsay Cedex, France \\ danielle.hilhorst@math.u-psud.fr \\ JOHANNES KAMPMANN \\ Fakultät für Mathematik, Universität Regensburg \\ 93040, Regensburg, Germany \\ johannes.kampmann@mathematik.uni-regensburg.de \\ THANH NAM NGUYEN \\ CNRS, Laboratoire de Mathématique, Université de Paris-Sud \\ F-91405 Orsay Cedex, France \\ thanh-nam.nguyen@math.u-psud.fr \\ KRISTOFFER GEORGE VAN DER ZEE \\ School of Mathematical Sciences, The University of Nottingham \\ University Park, Nottingham NG7 2RD, United Kingdom \\ kg.vanderzee@nottingham.ac.uk \\ Received 28 April 2014 \\ Communicated by (xxxxxxxxxx)
}

\begin{abstract}
We consider a diffuse-interface tumor-growth model which has the form of a phase-field system. We characterize the singular limit of this problem. More precisely, we formally prove that as the coefficient of the reaction term tends to infinity, the solution converges to the solution of a novel free boundary problem. We present numerical simulations which illustrate the convergence of the diffuse-interface model to the identified sharp-interface limit.
\end{abstract}

Keywords: Reaction-diffusion system; singular perturbation; interface motion; matched asymptotic expansion; tumor-growth model; phase-field model; gradient flow; stabilized Crank-Nicolson method; convex-splitting scheme

AMS Subject Classification: 35B40, 35K57, 35R35, 92B99

\section{Introduction}

\subsection{Diffuse-interface tumor-growth models}

Diffuse-interface tumor-growth models have been studied recently in several articles. ${ }^{39,18,28,27,29}$ We refer to the reviews in Refs. $17,19,33$. The basic model is 
composed of a fourth order parabolic equation for the tumor cell phase $u: \Omega \rightarrow \mathbb{R}$ coupled to an elliptic equation for the nutrient phase $\sigma: \Omega \rightarrow \mathbb{R}$ :

$$
\begin{aligned}
u_{t} & =\Delta\left(-\varepsilon^{-1} f(u)-\varepsilon \Delta u\right)+\varepsilon^{-1} p_{0} \sigma u \\
0 & =\Delta \sigma-\varepsilon^{-1} p_{0} \sigma u
\end{aligned}
$$

where $\varepsilon^{2}$ is the diffusivity corresponding to the surface energy, the positive constant $p_{0}$ is a proliferation growth parameter, and $f$ is a bistable function.

Introducing the chemical potential $\mu: \Omega \rightarrow \mathbb{R}$, given by

$$
\mu:=-\varepsilon^{-1} f(u)-\varepsilon \Delta u,
$$

(1.1a)-(1.1b) becomes

$$
\begin{aligned}
u_{t} & =\Delta \mu+\varepsilon^{-1} p_{0} \sigma u, \\
\mu & =-\varepsilon^{-1} f(u)-\varepsilon \Delta u, \\
0 & =\Delta \sigma-\varepsilon^{-1} p_{0} \sigma u .
\end{aligned}
$$

The above system models the evolution of the first stage of a growing tumor. ${ }^{35}$ In this stage a tumor grows because of the consumption of nutrients that diffuse through the surrounding tissue. This stage is referred to as avascular growth, as the tumor has not yet acquired its own blood supply to nurture itself. Consumption of nutrients is modeled in (1.2a) and (1.2c) via the reactive terms. To describe the evolution of the tumor boundary a diffuse-interface description is employed. This is classically modeled in (1.2a) with a diffusion via the chemical potential $\mu$ which depends in a nonlinear manner on $u$ and contains the higher-order regularization $\varepsilon \Delta u$, see $(1.2 \mathrm{~b})$.

Diffuse-interface tumor-growth models fall within the broader class of multiconstituent tumor-growth models based on continuum mixture theory. ${ }^{4,9,6}$ The derivation of diffuse-interface models within continuum mixture theory has been reviewed in Ref. 33, and requires the set up of balance laws for each constituent as well as the specification of constraints on the constitutive choices imposed by the second law of thermodynamics. Typically, only the cellular and fluidic constituents of a tumor are modeled as parts of a mixture, while nutrients are considered separately. Recently however, a diffuse-interface tumor growth model has been proposed that incorporates all constituents within the mixture and is proven to be thermodynamically consistent; see Ref. 28. In fact, the model is of gradient-flow type.

The model from Ref. 28 is a modification of (1.2) and it is given by:

$$
\begin{aligned}
u_{t} & =\Delta \mu+\varepsilon^{-1} p(u)(\sigma-\delta \mu) \\
\mu & =-\varepsilon^{-1} f(u)-\varepsilon \Delta u \\
\sigma_{t} & =\Delta \sigma-\varepsilon^{-1} p(u)(\sigma-\delta \mu)
\end{aligned}
$$

where $\delta>0$ is a small regularization parameter, and the growth function $p(u)$ is 


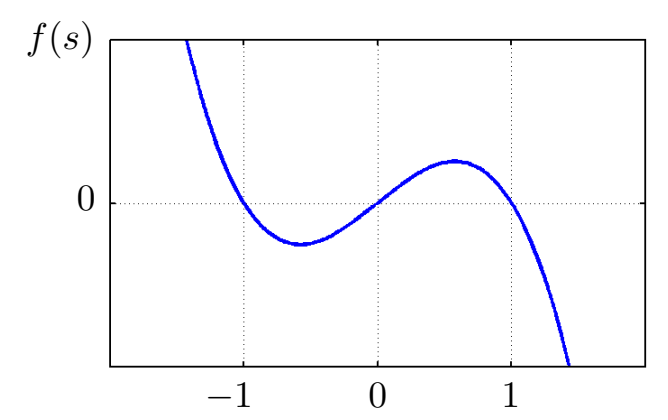

Fig. 1. Example of the bistable function $f(s)$.

defined by

$$
p(u):= \begin{cases}2 p_{0} \sqrt{W(u)} & u \in[-1,1] \\ 0 & \text { elsewhere. }\end{cases}
$$

Here $W(u):=-\int_{-1}^{u} f(s) d s$ is the classical Cahn-Hilliard double well free-energy density. We assume that the bistable function $f(u)$ has two stable roots \pm 1 , an unstable root 0 and mean zero: $\int_{-1}^{1} f(s) d s=0$. See for an example Fig. 1 .

Note that in (1.3a)-(1.3c) we have chosen a particular space-time scaling dependent on $\varepsilon$, which in our opinion is one of the interesting cases. In particular, the reactive interaction between $u$ and $\sigma$ responsible for growth, the diffusion of $\sigma$, as well as curvature effects will be shown to survive in the limit. The current study is most likely very useful in the study of other possible scalings.

The above model has the following multi-constituent interpretation: a tumorous phase $u \approx 1$, a healthy cell phase $u \approx-1$, and nutrient-rich extracellular water phase $\sigma \geq 0$. We refer to Ref. 28 for the interpretation of $\sigma$ as part of a mixture.

Note that, compared to (1.2a)-(1.2c), the reactive terms have been modified to be thermodynamically consistent. They include a regularization part $\delta \mu$ and they have been localized to the interface (since $p(u)$ is nonzero if $u \in(-1,1)$ ); see Ref. 28 for more details. The unknown pair $(u, \sigma)$ is a dissipative gradient flow for the energy functional

$$
\mathcal{E}(u, \sigma):=\int_{\Omega}\left(\frac{\varepsilon}{2}|\nabla u|^{2}+\frac{1}{\varepsilon} W(u)+\frac{\sigma^{2}}{2 \delta}\right)
$$

We refer to Theorem 1.1 for the proof of this property in a slightly more general context.

Note that there are various scalings of interest (cf. for instance Ref. 12) and that the one chosen in the definition of $\mathcal{E}(u, \sigma)$ above is only one of them. Furthermore, an important extension of the above energy includes effects due to chemotaxis. We shall not consider chemotaxis in this work, as this deserves a completely dedicated study of its own. 
In this work, we shall be interested in the singular limit $\varepsilon \downarrow 0$ of (1.3a)(1.3c) together with homogeneous Neumann boundary conditions. We furthermore shall be interested in numerical simulations which validate the identified singular limit and which go beyond the assumptions underlying the theory. We note that many articles involving singular limits have appeared over the years $3,15,34,2,13$ including overviews ${ }^{25,32,31}$ and numerical studies ${ }^{24,14}$. Articles involving formal asymptotics $^{1,11,12}$ are of particular interest for the analysis in for this paper.

\subsection{The main results}

We have to deal with a fourth order equation, at least if we substitute (1.3b) into (1.3a), which is a generalization of the Cahn-Hilliard equation. In this form, it seems complicated to deduce the singular limit; on the other hand, it is quite standard to pass to the limit in the Allen-Cahn equation and thus in associated phase-field models as well. More precisely, one knows how to pass to the limit as $\varepsilon \rightarrow 0$ in the equation

$$
\alpha u_{t}=\Delta u+\frac{1}{\varepsilon^{2}}(f(u)+\varepsilon \mu),
$$

namely equation (1.5b) below (cf. section 3.2). This motivates us to first consider the corresponding phase field approximation (1.5a)-(1.5e) and pass to the limit as $\varepsilon \rightarrow 0$ in this problem. Setting $\alpha=0$ in the corresponding result then yields the limit of the original Cahn-Hilliard type system.

It will turn out that the problems in both cases $\alpha>0$ and $\alpha=0$ are gradient flows and that the corresponding limit problems as $\varepsilon \downarrow 0$ are also gradient flows. This will be discussed in the sequel.

In order to study the singular limit of Problem (1.3a)-(1.3c) as $\varepsilon \downarrow 0$, we therefore introduce the following phase-field model

$$
\begin{aligned}
\alpha \mu_{t}^{\varepsilon}+u_{t}^{\varepsilon} & =\Delta \mu^{\varepsilon}+\varepsilon^{-1} p\left(u^{\varepsilon}\right)\left(\sigma^{\varepsilon}-\delta \mu^{\varepsilon}\right) & & \text { in } \Omega \times(0,+\infty), \\
\varepsilon^{-1} \mu^{\varepsilon}-\alpha u_{t}^{\varepsilon} & =-\varepsilon^{-2} f\left(u^{\varepsilon}\right)-\Delta u^{\varepsilon} & & \text { in } \Omega \times(0,+\infty), \\
\sigma_{t}^{\varepsilon} & =\Delta \sigma^{\varepsilon}-\varepsilon^{-1} p\left(u^{\varepsilon}\right)\left(\sigma^{\varepsilon}-\delta \mu^{\varepsilon}\right) & & \text { in } \Omega \times(0,+\infty),
\end{aligned}
$$

together with the boundary and initial conditions

$$
\begin{array}{lll}
\frac{\partial \mu^{\varepsilon}}{\partial \nu}=\frac{\partial u^{\varepsilon}}{\partial \nu}=\frac{\partial \sigma^{\varepsilon}}{\partial \nu}=0 & \text { on } \partial \Omega \times(0,+\infty), \\
\alpha \mu^{\varepsilon}(\cdot, 0)=\alpha \mu_{0}^{\varepsilon}, \quad u^{\varepsilon}(\cdot, 0)=u_{0}^{\varepsilon}, & \sigma^{\varepsilon}(\cdot, 0)=\sigma_{0}^{\varepsilon}, & \text { on } \Omega .
\end{array}
$$

Here, $\Omega$ is a smooth bounded domain of $\mathbb{R}^{N}(N \geq 2), \nu$ is the outer unit normal vector to $\partial \Omega$ and $\alpha$ is a positive constant. We denote by $\left(P_{\varepsilon}\right)$ the problem (1.5a)-(1.5e). Setting $\alpha=0$ in the singular limit of Problem $\left(P_{\varepsilon}\right)$, we will obtain the singular limit of Problem (1.3a)-(1.3c). Problem $\left(P_{\varepsilon}\right)$ possesses the Lyapunov functional

$$
E_{\varepsilon}(u, \mu, \sigma):=\int_{\Omega}\left(\frac{\varepsilon}{2}|\nabla u|^{2}+\frac{1}{\varepsilon} W(u)+\frac{\alpha \mu^{2}}{2}+\frac{\sigma^{2}}{2 \delta}\right) .
$$


We will prove in section 2 that Problem $\left(P_{\varepsilon}\right)$ is a gradient flow associated to the functional $E_{\varepsilon}(u, \mu, \sigma)$.

Theorem 1.1. Let $\left(u^{\varepsilon}, \mu^{\varepsilon}, \sigma^{\varepsilon}\right)$ be a smooth solution of Problem $\left(P_{\varepsilon}\right)$. Then $E_{\varepsilon}\left(u^{\varepsilon}, \mu^{\varepsilon}, \sigma^{\varepsilon}\right)$ is decreasing along solution orbits.

We will show in the following that, if in some sense

$$
\mu^{\varepsilon} \longrightarrow \mu, \quad u^{\varepsilon} \longrightarrow u, \quad \sigma^{\varepsilon} \longrightarrow \sigma,
$$

then the triple $(\mu, u, \sigma)$ is characterized by a limit free boundary problem, where the interface motion equation appears as the limit of the equation (1.5b). A rigorous proof of the convergence of the solution of the equation (1.5b) may for instance be found in Ref. 1. According to Ref. 1, the function $u$ only takes the two values -1 or 1 and the interface which separates the regions where $\{u=-1\}$ and $\{u=1\}$ partially moves according to its mean curvature.

Assumption on initial conditions: We assume that as $\varepsilon \downarrow 0$,

$$
\mu_{0}^{\varepsilon} \longrightarrow \mu_{0}, \quad u_{0}^{\varepsilon} \longrightarrow u_{0}, \quad \sigma_{0}^{\varepsilon} \longrightarrow \sigma_{0},
$$

in some sense and that there exists a closed smooth hypersurface without boundary $\Gamma_{0} \subset \subset \Omega$ which divides $\Omega$ into two subdomains $\Omega^{+}(0)$ and $\Omega^{-}(0)$ such that

$$
u_{0}=\left\{\begin{array}{cc}
-1 & \text { in } \Omega^{-}(0), \\
1 & \text { in } \Omega^{+}(0) .
\end{array}\right.
$$

We also assume that $\Omega^{+}(0)$ is the region enclosed by $\Gamma_{0}$ and that $\Omega^{-}(0)$ is the region enclosed between $\partial \Omega$ and $\Gamma_{0}$.

Now, we are ready to introduce a free boundary problem namely the singular limit of Problem $\left(P_{\varepsilon}\right)$ as $\varepsilon \downarrow 0$ :

$$
\begin{aligned}
& u(x, t)= \begin{cases}1 & \text { in } \Omega^{+}(t), t \in(0, T] \\
-1 & \text { in } \Omega^{-}(t), t \in(0, T]\end{cases} \\
& \alpha V_{n}=-(N-1) \kappa+\mathcal{C} \mu \quad \text { on } \Gamma(t), t \in(0, T] \\
& \alpha \mu_{t}+u_{t}=\Delta \mu+2 \sqrt{2} p_{0}(\sigma-\delta \mu) \delta_{0}(x-\Gamma(t)) \quad \text { in } \Omega \times(0, T] \text {, } \\
& \sigma_{t}=\Delta \sigma-2 \sqrt{2} p_{0}(\sigma-\delta \mu) \delta_{0}(x-\Gamma(t)) \quad \text { in } \Omega \times(0, T],
\end{aligned}
$$

together with the boundary and initial conditions

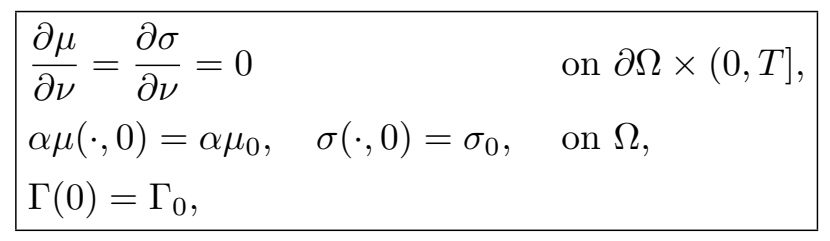


Here, $\Gamma(t) \subset \subset \Omega$ is a closed hypersurface; $\Omega^{+}(t)$ is the region enclosed by $\Gamma(t)$; $\Omega^{-}(t)=\Omega \backslash\left(\Omega^{+}(t) \cup \Gamma(t)\right) ; \delta_{0}$ is the Dirac distribution; $V_{n}: \Gamma(t) \rightarrow \mathbb{R}^{N}$ is the normal velocity of the evolving interface $\Gamma(t), \kappa$ is the mean curvature at each point of $\Gamma(t)$ and

$$
\mathcal{C}=\left[\int_{-1}^{1} \sqrt{W(s) / 2} \mathrm{~d} s\right]^{-1} .
$$

We denote by $\left(P_{0}\right)$ the problem $(1.7 \mathrm{a})-(1.7 \mathrm{~g})$ and define

$$
\Gamma_{T}:=\bigcup_{t \in(0, T]} \Gamma(t) \times\{t\}
$$

Definition 1.1. We say that the triple $\left(\Gamma_{T}, \mu, \sigma\right)$ is a solution of Problem $\left(P_{0}\right)$ if

(1) the set $\bigcup_{0 \leq t \leq T} \Gamma(t) \times\{t\}$ is smooth, namely $\Gamma(t)$ is a smooth hypersurface which lies entirely within $\Omega$ for all $t \in[0, T]$ and $\Gamma(t)$ varies smoothly in time;

(2) for all test functions $\psi \in \mathcal{F}_{T}:=\left\{\psi \in C^{2,1}(\bar{\Omega} \times[0, T])\right.$ such that $\frac{\partial \psi}{\partial \nu}=0$ on $\partial \Omega \times[0, T]$ and $\left.\psi(T)=0\right\}$, we have

$$
\begin{aligned}
\int_{0}^{T} \int_{\Omega}(-\alpha \mu-u) \psi_{t}-\int_{\Omega}( & \left.\alpha \mu_{0}+u_{0}\right) \psi(0) \\
& =\int_{0}^{T} \int_{\Omega} \mu \Delta \psi+2 \sqrt{2} p_{0} \int_{0}^{T} \int_{\Gamma(t)}(\sigma-\delta \mu) \psi,
\end{aligned}
$$

and

$$
\int_{0}^{T} \int_{\Omega}-\sigma \psi_{t}-\int_{\Omega} \sigma_{0} \psi(0)=\int_{0}^{T} \int_{\Omega} \sigma \Delta \psi-2 \sqrt{2} p_{0} \int_{0}^{T} \int_{\Gamma(t)}(\sigma-\delta \mu) \psi .
$$

Now, in order to state the next result, we need some notations. Let $n^{+}(t), n^{-}(t)$ be the outer unit normal vectors to $\partial \Omega^{+}(t)$ and $\partial \Omega^{-}(t)$, respectively. Note that $n^{+}=-n^{-}$on $\Gamma_{T}$, so we may define $n:=n^{+}=-n^{-}$on $\Gamma_{T}$. We define $\llbracket \cdot \rrbracket$ the jump across $\Gamma(t)$, by $\llbracket \phi \rrbracket:=\phi^{+}-\phi^{-}$, where $\phi^{ \pm}$should be understood as the following limit

$$
\phi^{ \pm}(\cdot):=\lim _{\rho \rightarrow 0^{-}} \phi\left(\cdot+\rho n^{ \pm}(t)\right) \text { on } \Gamma(t)
$$

We also define

$$
Q_{T}^{+}:=\bigcup_{t \in(0, T]} \Omega^{+}(t) \times\{t\}, \quad \text { and } \quad Q_{T}^{-}:=\bigcup_{t \in(0, T]} \Omega^{-}(t) \times\{t\} .
$$


Theorem 1.2. Assume that Problem $\left(P_{0}\right)$ possesses a solution $\left(\Gamma_{T}, \mu, \sigma\right)$ such that $\Gamma_{T}$ is smooth on the time interval $(0, T)$ and that $\mu$ and $\sigma$ are smooth up to $\Gamma_{T}$ on both sides of $\Gamma_{T}$. Then the triple $\left(\Gamma_{T}, \mu, \sigma\right)$ satisfies:

$\begin{aligned} \alpha \mu_{t} & =\Delta \mu & & \text { in } Q_{T}^{+} \cup Q_{T}^{-}, \\ \sigma_{t} & =\Delta \sigma & & \text { on } Q_{T}^{+} \cup Q_{T}^{-}, \\ \llbracket \mu \rrbracket & =\llbracket \sigma \rrbracket=0 & & \text { on } \Gamma_{T}, \\ \llbracket \frac{\partial \mu}{\partial n} \rrbracket & =-2 V_{n}+2 \sqrt{2} p_{0}(\sigma-\delta \mu) & & \text { on } \Gamma_{T}, \\ \llbracket \frac{\partial \sigma}{\partial n} \rrbracket & =-2 \sqrt{2} p_{0}(\sigma-\delta \mu) & & \text { on } \Gamma_{T}, \\ \alpha V_{n} & =-(N-1) \kappa+\mathcal{C} \mu & & \text { on } \Gamma_{T},\end{aligned}$

together with the boundary and initial conditions

$$
\begin{array}{ll}
\frac{\partial \mu}{\partial \nu}=\frac{\partial \sigma}{\partial \nu}=0 & \text { on } \partial \Omega \times(0, T], \\
\mu(\cdot, 0)=\mu_{0}, \quad \sigma(\cdot, 0)=\sigma_{0}, & \text { on } \Omega, \\
\Gamma(0)=\Gamma_{0} . &
\end{array}
$$

In this case, we say that $\left(\Gamma_{T}, \mu, \sigma\right)$ is a classical solution of Problem $\left(P_{0}\right)$ on the time interval $[0, T]$.

Problem $\left(P_{0}\right)$ possesses the Lyapunov functional

$$
E(\Gamma, \mu, \sigma):=\frac{2}{\mathcal{C}} \int_{\Gamma} 1 \mathrm{~d} \Gamma+\int_{\Omega}\left(\frac{\alpha \mu^{2}}{2}+\frac{\sigma^{2}}{2 \delta}\right),
$$

which is analogous to the Lyapunov functional satisfied by Problem $\left(P_{\varepsilon}\right)$.

Theorem 1.3. Let $\left(\Gamma_{T}, \mu, \sigma\right)$ be a classical solution of Problem $\left(P_{0}\right)$. Then $E(\Gamma, \mu, \sigma)$ is decreasing along solution orbits.

Finally, we will formally prove the following result.

Theorem 1.4. Let $\left(\mu^{\varepsilon}, u^{\varepsilon}, \sigma^{\varepsilon}\right)$ be solution of Problem $\left(P_{\varepsilon}\right)$. We suppose that Problem $\left(P_{0}\right)$ possesses a unique classical solution on the interval $[0, T]$. If $\varepsilon \rightarrow 0$,

$$
\mu^{\varepsilon} \longrightarrow \mu, \quad u^{\varepsilon} \longrightarrow u, \quad \sigma^{\varepsilon} \longrightarrow \sigma \text { in a strong enough sense, }
$$

then $\left(\Gamma_{T}, \mu, \sigma\right)$ coincide with the classical solution of Problem $\left(P_{0}\right)$ and $u$ is given by (1.7a).

We note that the singular limit corresponds to a moving boundary problem which is similar to other sharp-interface tumor-growth models. ${ }^{26,8,20,7,10,39,18}$ The interesting characteristic of the current singular limit is that the reactive terms of the phase-field model collapse to the interface in the limit, which is different than in some other models where the reactive terms remain as bulk contributions. 
The current identified limit is consistent with the multi-constituent mixture interpretation. Indeed, in the diffuse-interface model, the tumor-cell concentration and the healthy-cell concentration add up to a fixed cell concentration: pure tumor cells where $u=1$, pure healthy cells where $u=-1$, and a mixture at diffuse interfaces. ${ }^{28}$ This means that in pure phases we have a full single-constituent cell concentration. As a consequence, in a pure tumor phase the tumor concentration can not increase further. Tumor growth thus happens within the diffuse interface, where the tumor-cell concentration can increase at the cost of healthy cells (provided, of course, sufficient nutrients are available). Upon collapsing the thickness of the diffuse interface, one thus expects a Dirac-delta term at the interface to be responsible for front propagation.

The moving boundary problem (1.9) may be simplified by setting some parameters to zero or infinity (formally). In particular, two interesting simplifications are possible. The first one is when $\Gamma(t)$ propagates because of nonzero $\sigma$ at $\Gamma(t)$, and $\sigma$ satisfies a steady interface problem. This is a so-called tumor-front propagation with quasi-steady nutrient evolution. The second one is when $\Gamma(t)$ propagates simply because of a constant nonzero $\sigma=\sigma_{0}$. These two simplifications allow for direct comparisons with numerical simulations of the diffuse-interface model. We will take up such a comparison in Section 4 with 2-D and 3-D numerical simulations using the energy-stable scheme from Ref. 40. We verify the convergence of the diffuseinterface model to its sharp-interface limit, and explore a situation with topological changes which is not covered by Theorem 1.2.

The remainder of the paper is organized as follows: In Section 2 we prove Theorem 1.1, Theorem 1.2 and Theorem 1.3; In Section 3, we formally justify Theorem 1.4. Finally in Section 4 we present numerical experiments.

\section{Proof of the main results}

\subsection{Proof of Theorem 1.1}

It is sufficient to prove that

$$
\frac{d}{d t} E_{\varepsilon}\left(u^{\varepsilon}, \mu^{\varepsilon}, \sigma^{\varepsilon}\right) \leq 0
$$


For simplicity, we write $u, \mu, \sigma$ instead of $u^{\varepsilon}, \mu^{\varepsilon}, \sigma^{\varepsilon}$. Now, the inequality (2.1) follows from the following computations:

$$
\begin{aligned}
\frac{d}{d t} E_{\varepsilon}(u, \mu, \sigma)= & \int_{\Omega}\left(\varepsilon \nabla u \nabla u_{t}+\varepsilon^{-1} W^{\prime}(u) u_{t}+\alpha \mu \mu_{t}+\frac{\sigma \sigma_{t}}{\delta}\right) \\
= & \int_{\Omega}\left(\left[-\varepsilon^{-1} f(u)-\varepsilon \Delta u\right] u_{t}+\alpha \mu \mu_{t}+\frac{\sigma \sigma_{t}}{\delta}\right) \\
= & \int_{\Omega}\left(\left(\mu-\alpha \varepsilon u_{t}\right) u_{t}+\alpha \mu \mu_{t}\right)+\int_{\Omega} \frac{\sigma}{\delta}\left(\Delta \sigma-\varepsilon^{-1} p(u)(\sigma-\delta \mu)\right) \\
= & -\int_{\Omega} \alpha \varepsilon u_{t}^{2}+\int_{\Omega} \mu\left(u_{t}+\alpha \mu_{t}\right)+\int_{\Omega} \frac{\sigma}{\delta}\left(\Delta \sigma-\varepsilon^{-1} p(u)(\sigma-\delta \mu)\right) \\
= & -\int_{\Omega} \alpha \varepsilon u_{t}^{2}+\int_{\Omega} \mu\left(\Delta \mu+\varepsilon^{-1} p(u)(\sigma-\delta \mu)\right) \\
& +\int_{\Omega} \frac{\sigma}{\delta}\left(\Delta \sigma-\varepsilon^{-1} p(u)(\sigma-\delta \mu)\right) \\
= & -\int_{\Omega} \alpha \varepsilon u_{t}^{2}-\int_{\Omega}|\nabla \mu|^{2}-\int_{\Omega} \frac{|\nabla \sigma|^{2}}{\delta} \\
& +\varepsilon^{-1} \int_{\Omega} p(u)\left(\mu(\sigma-\delta \mu)-\frac{\sigma}{\delta}(\sigma-\delta \mu)\right) \\
= & -\int_{\Omega} \alpha \varepsilon u_{t}^{2}-\int_{\Omega}|\nabla \mu|^{2}-\int_{\Omega} \frac{|\nabla \sigma|^{2}}{\delta}-\varepsilon^{-1} \int_{\Omega} p(u)\left(\sqrt{\delta} \mu-\frac{\sigma}{\sqrt{\delta}}\right)^{2} \\
\leq & 0 .
\end{aligned}
$$

\subsection{Proof of Theorem 1.2}

First, we recall that $n^{+}(t), n^{-}(t)$ are the outer unit normal vectors to $\partial \Omega^{+}(t)$ and $\partial \Omega^{-}(t)$, respectively and $n:=n^{+}=-n^{-}$on $\Gamma_{T}$. We define $V_{n}=V \cdot n_{+}$, where $V$ is the velocity of displacement of the interface $\Gamma_{T}$.

\subsubsection{Equations for $\mu$}

We recall that $u, \mu$ satisfy

$$
\begin{aligned}
\int_{0}^{T} \int_{\Omega}(-\alpha \mu-u) \psi_{t}-\int_{\Omega}\left(\alpha \mu_{0}\right. & \left.+u_{0}\right) \psi(0) \\
& =\int_{0}^{T} \int_{\Omega} \mu \Delta \psi+2 \sqrt{2} p_{0} \int_{0}^{T} \int_{\Gamma(t)}(\sigma-\delta \mu) \psi,
\end{aligned}
$$

for all $\psi \in \mathcal{F}_{T}$. We define the terms $A_{1}, A_{2}$ and the diffusion term $B$ by

$$
A_{1}:=\int_{0}^{T} \int_{\Omega}-\alpha \mu \psi_{t}, \quad A_{2}:=\int_{0}^{T} \int_{\Omega}-u \psi_{t}, \quad \text { and } \quad B:=\int_{0}^{T} \int_{\Omega} \mu \Delta \psi .
$$


Analysis of the terms $\mathbf{A}_{\mathbf{1}}$ and $\mathbf{A}_{\mathbf{2}}$ : Our analysis of the terms $A_{1}$ and $A_{2}$ relies on the Reynolds transport theorem, by which we have

$$
\frac{\mathrm{d}}{\mathrm{d} t} \int_{\Omega^{ \pm}(t)} \phi \psi=\int_{\Omega^{ \pm}(t)}\left(\phi_{t} \psi+\phi \psi_{t}\right) \pm \int_{\Gamma(t)} V_{n} \phi^{ \pm} \psi,
$$

for all smooth function $\psi$ and for function $\phi$. These equations for the integrals over $\Omega^{ \pm}(t)$ yield

$$
\frac{\mathrm{d}}{\mathrm{d} t} \int_{\Omega^{+}(t) \cup \Omega^{-}(t)} \phi \psi=\int_{\Omega^{+}(t) \cup \Omega^{-}(t)}\left(\phi_{t} \psi+\phi \psi_{t}\right)+\int_{\Gamma(t)} V_{n} \llbracket \phi \rrbracket \psi .
$$

Hence we have

$$
\int_{\Omega^{+}(t) \cup \Omega^{-}(t)}-\phi \psi_{t}=\int_{\Omega^{+}(t) \cup \Omega^{-}(t)} \phi_{t} \psi+\int_{\Gamma(t)} V_{n} \llbracket \phi \rrbracket \psi-\frac{\mathrm{d}}{\mathrm{d} t} \int_{\Omega^{+}(t) \cup \Omega^{-}(t)} \phi \psi .
$$

In our case, we choose $\phi:=\alpha \mu$ in (2.3) and integrate from 0 to $T$. This yields

$$
\begin{aligned}
A_{1} & =\int_{0}^{T} \int_{\Omega^{+}(t) \cup \Omega^{-}(t)} \alpha \mu_{t} \psi+\int_{0}^{T} \int_{\Gamma(t)} V_{n} \llbracket \alpha \mu \rrbracket \psi-\int_{0}^{T} \frac{\mathrm{d}}{\mathrm{d} t} \int_{\Omega^{+}(t) \cup \Omega^{-}(t)} \alpha \mu \psi \\
& =\int_{0}^{T} \int_{\Omega^{+}(t) \cup \Omega^{-}(t)} \alpha \mu_{t} \psi+\int_{0}^{T} \int_{\Gamma(t)} \alpha V_{n} \llbracket \mu \rrbracket \psi+\int_{\Omega^{+}(0) \cup \Omega^{-}(0)} \alpha \mu(0) \psi(0) .
\end{aligned}
$$

Similarly, we apply the formula (2.3) for $\phi:=u$ to obtain

$$
\begin{aligned}
A_{2} & =\int_{0}^{T} \int_{\Omega^{+}(t) \cup \Omega^{-}(t)} u_{t} \psi+\int_{0}^{T} \int_{\Gamma(t)} V_{n} \llbracket u \rrbracket \psi+\int_{\Omega^{+}(0) \cup \Omega^{-}(0)} u(0) \psi(0) \\
& =2 \int_{0}^{T} \int_{\Gamma(t)} V_{n} \psi+\int_{\Omega^{+}(0) \cup \Omega^{-}(0)} u(0) \psi(0) .
\end{aligned}
$$

Analysis of the term B: We write $B$ as the sum

$$
B=\int_{0}^{T}\left(\int_{\Omega^{+}(t)} \mu \Delta \psi+\int_{\Omega^{-}(t)} \mu \Delta \psi\right) .
$$

Integration by parts yields

$$
\begin{aligned}
\int_{\Omega^{-}(t)}^{\mu \Delta \psi} & =-\int_{\Omega^{-}(t)} \nabla \mu \nabla \psi+\int_{\Gamma(t)} \frac{\partial \psi}{\partial n^{-}} \mu^{-} \\
& =\int_{\Omega^{-}(t)} \Delta \mu \psi-\int_{\Gamma(t)} \frac{\partial \mu^{-}}{\partial n^{-}} \psi-\int_{\partial \Omega} \frac{\partial \mu}{\partial \nu} \psi+\int_{\Gamma(t)} \frac{\partial \psi}{\partial n^{-}} \mu^{-} \\
& =\int_{\Omega^{-}(t)} \Delta \mu \psi+\int_{\Gamma(t)} \frac{\partial \mu^{-}}{\partial n} \psi-\int_{\partial \Omega} \frac{\partial \mu}{\partial \nu} \psi-\int_{\Gamma(t)} \frac{\partial \psi}{\partial n} \mu^{-}
\end{aligned}
$$

and

$$
\int_{\Omega^{+}(t)} \mu \Delta \psi=\int_{\Omega^{+}(t)} \Delta \mu \psi-\int_{\Gamma(t)} \frac{\partial \mu^{+}}{\partial n} \psi+\int_{\Gamma(t)} \frac{\partial \psi}{\partial n} \mu^{+}
$$

which implies that

$$
\int_{\Omega^{+}(t) \cup \Omega^{-}(t)} \mu \Delta \psi=\int_{\Omega^{+}(t) \cup \Omega^{-}(t)} \Delta \mu \psi-\int_{\Gamma(t)} \llbracket \frac{\partial \mu}{\partial n} \rrbracket \psi+\int_{\Gamma(t)} \frac{\partial \psi}{\partial n} \llbracket \mu \rrbracket-\int_{\partial \Omega} \frac{\partial \mu}{\partial \nu} \psi .
$$


Integrating this identity from 0 to $T$, we obtain

$$
\begin{aligned}
B=\int_{0}^{T} \int_{\Omega^{+}(t) \cup \Omega^{-}(t)} & \Delta \mu \psi \\
& -\int_{0}^{T} \int_{\Gamma(t)} \llbracket \frac{\partial \mu}{\partial n} \rrbracket \psi+\int_{0}^{T} \int_{\Gamma(t)} \frac{\partial \psi}{\partial n} \llbracket \mu \rrbracket-\int_{0}^{T} \int_{\partial \Omega} \frac{\partial \mu}{\partial \nu} \psi .
\end{aligned}
$$

Conclusion: Combining (2.2), (2.4) (2.5) and (2.6), we then have for all $\psi \in \mathcal{F}_{T}$,

$$
\begin{aligned}
& \int_{0}^{T} \int_{\Omega^{+}(t) \cup \Omega^{-}(t)} \alpha \mu_{t} \psi+\int_{0}^{T} \int_{\Gamma(t)} V_{n}(\alpha \llbracket \mu \rrbracket+2) \psi \\
& +\int_{\Omega^{+}(t) \cup \Omega^{-}(t)} \alpha\left(\mu(0)-\mu_{0}\right) \psi(0)+\int_{\Omega^{+}(t) \cup \Omega^{-}(t)}\left(u(0)-u_{0}\right) \psi(0) \\
& =\int_{0}^{T} \int_{\Omega^{+}(t) \cup \Omega^{-}(t)} \Delta \mu \psi-\int_{0}^{T} \int_{\Gamma(t)} \llbracket \frac{\partial \mu}{\partial n} \rrbracket \psi+\int_{0}^{T} \int_{\Gamma(t)} \frac{\partial \psi}{\partial n} \llbracket \mu \rrbracket-\int_{0}^{T} \int_{\partial \Omega} \frac{\partial \mu}{\partial \nu} \psi \\
& +\int_{0}^{T} \int_{\Gamma(t)} 2 \sqrt{2} p_{0}(\sigma-\delta \mu) \psi
\end{aligned}
$$

By using test functions with suitable supports, namely $\psi \in C_{0}^{\infty}\left(Q_{T}^{+}\right)$and $\psi \in$ $C_{0}^{\infty}\left(Q_{T}^{-}\right)$, we deduce that

$$
\alpha \mu_{t}=\Delta \mu \text { in } Q_{T}^{+} \cup Q_{T}^{-} .
$$

Similarly, by taking $\psi \in C_{0}^{\infty}\left(Q_{T}\right)$ such that $\frac{\partial \psi}{\partial n}=0$ on $\Gamma_{T}$ (we refer to Remark 2.1 below for the construction of such functions), we obtain

$$
V_{n}(2+\alpha \llbracket \mu \rrbracket)=-\llbracket \frac{\partial \mu}{\partial n} \rrbracket+2 \sqrt{2} p_{0}(\sigma-\delta \mu) \text { on } \Gamma_{T} .
$$

Now, we take $\psi \in C_{0}^{\infty}\left(Q_{T}\right)$ to deduce that

$$
\int_{0}^{T} \int_{\Gamma(t)} \frac{\partial \psi}{\partial n} \llbracket \mu \rrbracket \quad=0 \text { for all } \psi \in C_{0}^{\infty}\left(Q_{T}\right)
$$

Therefore,

$$
\llbracket \mu \rrbracket=0 \text { on } \Gamma_{T} .
$$

It follows from (2.9) and (2.11) that

$$
2 V_{n}=-\llbracket \frac{\partial \mu}{\partial n} \rrbracket+2 \sqrt{2} p_{0}(\sigma-\delta \mu) \quad \text { on } \Gamma_{T} .
$$

Now, for the initial conditions, we use the test function $\psi \in \mathcal{F}_{T}$ such that $\psi=0$ on $\partial \Omega \times(0, T)$ to obtain

$$
u(0)+\alpha \mu(0)=u_{0}+\alpha \mu_{0},
$$

which in view of (1.6) implies that

$$
\mu(0)=\mu_{0}, \quad u(0)=u_{0}
$$


Finally, the remaining term in (2.7) allows us to conclude that

Therefore, $\mu$ satisfies the equations:

$$
\frac{\partial \mu}{\partial \nu}=0
$$

$$
\begin{aligned}
& \alpha \mu_{t}=\Delta \mu \quad \text { in } Q_{T}^{+} \cup Q_{T}^{-}, \\
& 2 V_{n}=-\llbracket \frac{\partial \mu}{\partial n} \rrbracket+2 \sqrt{2} p_{0}(\sigma-\delta \mu) \quad \text { on } \Gamma_{T}, \\
& \llbracket \mu \rrbracket=0 \quad \text { on } \Gamma_{T},
\end{aligned}
$$

together with the boundary condition and the initial condition:

$$
\frac{\partial \mu}{\partial \nu}=0, \quad \mu(0)=\mu_{0}
$$

Remark 2.1. Let $\widetilde{\psi} \in C_{0}^{\infty}\left(Q_{T}\right)$. In the following, we construct a function $\psi \in$ $C_{0}^{\infty}\left(Q_{T}\right)$ such that

$$
\left.\frac{\partial \psi}{\partial n}\right|_{\Gamma_{T}}=0 \quad \text { and } \psi=\widetilde{\psi} \quad \text { on } \quad \Gamma_{T}
$$

Let $\widetilde{d}(x, t)$ be the signed distance function to $\Gamma(t)$ (see (3.3) below). Since $\bigcup_{0 \leq t \leq T} \Gamma(t) \times\{t\}$ is smooth, there exists $\delta$ small enough such that $\widetilde{d}(x, t)$ is smooth in

$$
\mathcal{V}:=\{(x, t) \in \Omega \times[0, T],|\widetilde{d}(x, t)|<\delta\}
$$

moreover for all $(x, t) \in \mathcal{V}$ there exists a unique $(y, t)$ in $\Gamma(t) \times\{t\}$ such that $|\widetilde{d}(x, t)|=|x-y|$ and $(\nabla \widetilde{d}(x, t), t)=n(y, t)$. More precisely,

$$
(y, t)=J(x, t):=(x-\nabla \widetilde{d}(x, t) \widetilde{d}(x, t), t),
$$

where the projection operator $J$ is a smooth map from $\mathcal{V}$ into $\mathbb{R}^{N+1}$. We define $\psi$ on $\mathcal{V}$ by

$$
\psi(\cdot):=\widetilde{\psi}(\mathcal{J}(\cdot)) .
$$

Then $\psi$ is smooth on $\mathcal{V}$ and (2.12) holds. Moreover, we can extend $\psi$ to a smooth function on $Q_{T}$.

\subsubsection{Equations for $\sigma$}

Since the computations in this section are similar to the previous ones, we will only give a sketch of the necessary steps. For $\psi \in C_{0}^{\infty}\left(Q_{T}\right)$, we have

$$
\int_{0}^{T} \int_{\Omega}-\sigma \psi_{t}=\int_{0}^{T} \int_{\Omega} \sigma \Delta \psi-2 \sqrt{2} p_{0} \int_{0}^{T} \int_{\Gamma(t)}(\sigma-\delta \mu) \psi
$$

We define two terms

$$
C:=\int_{0}^{T} \int_{\Omega}-\sigma \psi_{t} \text { and } D:=\int_{0}^{T} \int_{\Omega} \sigma \Delta \psi
$$


One can easily deduce that

$$
C=\int_{0}^{T} \int_{\Omega^{+}(t) \cup \Omega^{-}(t)} \sigma_{t} \psi+\int_{0}^{T} \int_{\Gamma(t)} V_{n} \llbracket \sigma \rrbracket \psi,
$$

and

$$
D=\int_{0}^{T} \int_{\Omega^{+}(t) \cup \Omega^{-}(t)} \Delta \sigma \psi-\int_{0}^{T} \int_{\Gamma(t)} \llbracket \frac{\partial \sigma}{\partial n} \rrbracket \psi+\int_{0}^{T} \int_{\Gamma(t)} \frac{\partial \psi}{\partial n} \llbracket \sigma \rrbracket .
$$

It follows that

$$
\begin{gathered}
\int_{0}^{T} \int_{\Omega^{+}(t) \cup \Omega^{-}(t)} \sigma_{t} \psi+\int_{0}^{T} \int_{\Gamma(t)} V_{n} \llbracket \sigma \rrbracket \psi=\int_{0}^{T} \int_{\Omega^{+}(t) \cup \Omega^{-}(t)} \Delta \sigma \psi \\
-\int_{0}^{T} \int_{\Gamma(t)} \llbracket \frac{\partial \sigma}{\partial n} \rrbracket \psi+\int_{0}^{T} \int_{\Gamma(t)} \frac{\partial \psi}{\partial n} \llbracket \sigma \rrbracket-2 \sqrt{2} p_{0} \int_{0}^{T} \int_{\Gamma(t)}(\sigma-\delta \mu) \psi .
\end{gathered}
$$

and hence we have

$$
\begin{aligned}
\sigma_{t} & =\Delta \sigma & & \text { in } Q_{T}^{+} \cup Q_{T}^{-}, \\
\llbracket \frac{\partial \sigma}{\partial n} \rrbracket & =-2 \sqrt{2} p_{0}(\sigma-\delta \mu) & & \text { on } \Gamma_{T}, \\
\llbracket \sigma \rrbracket & =0 & & \text { on } \Gamma_{T},
\end{aligned}
$$

This concludes the proof of Theorem 1.2

\subsection{Proof of Theorem 1.3}

We prove below that

$$
\frac{d}{d t} E(\Gamma, \mu, \sigma) \leq 0
$$


The inequality (2.14) follows from the following computations and Theorem 4.3 p.355 and (4.12) p.356 in Ref. 21 :

$$
\begin{aligned}
\frac{d}{d t} E & (\Gamma, \mu, \sigma) \\
= & \frac{2}{\mathcal{C}} \int_{\Gamma}(N-1) \kappa V_{n}+\int_{\Omega^{+} \cup \Omega^{-}}\left(\alpha \mu \mu_{t}+\delta^{-1} \sigma \sigma_{t}\right) \\
= & \frac{2}{\mathcal{C}} \int_{\Gamma}\left[\mathcal{C} \mu-\alpha V_{n}\right] V_{n}+\int_{\Omega^{+} \cup \Omega^{-}}\left(\alpha \mu \mu_{t}+\delta^{-1} \sigma \sigma_{t}\right) \\
= & \int_{\Gamma} 2 \mu V_{n}-\int_{\Gamma} \frac{2 \alpha}{\mathcal{C}} V_{n}^{2}+\int_{\Omega^{+} \cup \Omega^{-}}\left(\mu \Delta \mu+\delta^{-1} \sigma \Delta \sigma\right) \\
= & \int_{\Gamma} \mu\left(-\llbracket \frac{\partial \mu}{\partial n} \rrbracket+2 \sqrt{2} p_{0}(\sigma-\delta \mu)\right)-\int_{\Gamma} \frac{2 \alpha}{\mathcal{C}} V_{n}^{2}+\int_{\Omega^{+} \cup \Omega^{-}}\left(\mu \Delta \mu+\delta^{-1} \sigma \Delta \sigma\right) \\
= & \int_{\Gamma} 2 \sqrt{2} p_{0} \mu(\sigma-\delta \mu)-\int_{\Gamma} \frac{2 \alpha}{\mathcal{C}} V_{n}^{2}-\int_{\Omega^{+} \cup \Omega^{-}}|\nabla \mu|^{2}+\int_{\Omega^{+} \cup \Omega^{-}} \delta^{-1} \sigma \Delta \sigma \\
= & \int_{\Gamma} 2 \sqrt{2} p_{0} \mu(\sigma-\delta \mu)-\int_{\Gamma} \frac{2 \alpha}{\mathcal{C}} V_{n}^{2}-\int_{\Omega^{+} \cup \Omega^{-}}|\nabla \mu|^{2} \\
& \quad-\int_{\Omega^{+} \cup \Omega^{-}} \frac{|\nabla \sigma|^{2}}{\delta}+\int_{\Gamma} \delta^{-1} \sigma \llbracket \frac{\partial \sigma}{\partial n} \rrbracket
\end{aligned}
$$

which in turn implies that

$$
\begin{aligned}
\frac{d}{d t} & E(\Gamma, \mu, \sigma) \\
= & \int_{\Gamma} 2 \sqrt{2} p_{0}\left(\mu(\sigma-\delta \mu)-\delta^{-1} \sigma(\sigma-\delta \mu)\right) \\
& \quad-\int_{\Gamma} \frac{2 \alpha}{\mathcal{C}} V_{n}^{2}-\int_{\Omega^{+} \cup \Omega^{-}}|\nabla \mu|^{2}-\int_{\Omega^{+} \cup \Omega^{-}} \frac{|\nabla \sigma|^{2}}{\delta} \\
= & -\int_{\Gamma} \frac{2 \alpha}{\mathcal{C}} V_{n}^{2}-\int_{\Omega^{+} \cup \Omega^{-}}|\nabla \mu|^{2}-\int_{\Omega^{+} \cup \Omega^{-}} \frac{|\nabla \sigma|^{2}}{\delta}-\int_{\Gamma} 2 \sqrt{2} p_{0}\left(\sqrt{\delta} \mu-\frac{\sigma}{\sqrt{\delta}}\right)^{2} \\
\leq & 0 .
\end{aligned}
$$

\section{Formal derivation of Theorem 1.4}

This section is devoted to prove formally theorem 1.4. We shall derive in turn equations for $u, \Gamma(t), \mu, \sigma$.

\subsection{Equation for $u$}

First, we formally show that $u$ only takes two values \pm 1 . To that purpose, we rewrite Equation $(1.5 \mathrm{~b})$ in the form

$$
\alpha u_{t}^{\varepsilon}=\Delta u^{\varepsilon}+\varepsilon^{-2} f\left(u^{\varepsilon}\right)+\varepsilon^{-1} \mu^{\varepsilon} .
$$

By setting $\tau:=t / \varepsilon^{2}$, we obtain

$$
\alpha u_{\tau}^{\varepsilon}=\varepsilon^{2} \Delta u^{\varepsilon}+f\left(u^{\varepsilon}\right)+\varepsilon \mu^{\varepsilon} .
$$


When $\varepsilon$ is small, we neglect the effect of diffusion term $\varepsilon^{2} \Delta u^{\varepsilon}$ and of the term $\varepsilon \mu^{\varepsilon}$ with respect to the term $f\left(u^{\varepsilon}\right)$, which yields the ordinary differential equation

$$
\alpha \frac{d u^{\varepsilon}}{d \tau} \cong f\left(u^{\varepsilon}\right) .
$$

Note that $\tau \rightarrow \infty$ as $\varepsilon \rightarrow 0$. Remembering that \pm 1 are two stable zeros of this equation. We formally deduce that as $\varepsilon \downarrow 0$

$$
\begin{cases}u^{\varepsilon}(x, t) \text { approaches }-1 & \text { if } u^{\varepsilon}(x, 0)<0 \\ u^{\varepsilon}(x, t) \text { approaches } \quad 1 & \text { if } u^{\varepsilon}(x, 0)>0 .\end{cases}
$$

This implies that the function $u$ which is the limit of $u^{\varepsilon}$ only takes two values \pm 1 .

\subsection{Formal derivation of the interface equation}

We define

$$
\Omega^{-}(t)=\{x \in \Omega: u(x, t)=-1\}, \quad \Omega^{+}(t)=\{x \in \Omega: u(x, t)=1\},
$$

and

$$
\Gamma(t):=\Omega \backslash\left(\Omega^{-}(t) \cup \Omega^{+}(t)\right) .
$$

Since roughly speaking, the regions $\{u=-1\}$ and $\{u=1\}$ are the "limit" of the regions $\left\{u^{\varepsilon} \approx-1\right\}$ and $\left\{u^{\varepsilon} \approx 1\right\}$ as $\varepsilon \rightarrow 0, \Gamma(t)$ can be considered as the limit as $\varepsilon \rightarrow 0$ of $\Gamma^{\varepsilon}(t)$ which is the interface between the two regions

$$
\left\{x \in \Omega: \quad u^{\varepsilon}(x, t) \approx-1\right\} \quad \text { and } \quad\left\{x \in \Omega: \quad u^{\varepsilon}(x, t) \approx 1\right\} .
$$

We recall that 0 is an unstable equilibria of Equation (3.1), and define

$$
\Gamma^{\varepsilon}(t)=\left\{x \in \Omega: \quad u^{\varepsilon}(x, t)=0\right\} \quad \text { for each } t \geq 0 .
$$

In what follows, we will use a formal asymptotic expansion to derive the equation describing $\Gamma(t)$. We need some preparations.

1. Signed distance function: We assume that the interface $\Gamma(t)$ is a smooth, closed hypersurface without boundary of $\mathbb{R}^{N}$. Further, we suppose that $\Omega^{+}(t)$ is the region enclosed by $\Gamma(t)$ and that $\Omega^{-}(t)$ is the region enclosed between $\partial \Omega$ and $\Gamma(t)$. Let $\tilde{d}(x, t)$ be the signed distance function to $\Gamma(t)$ defined by

$$
\tilde{d}(x, t)= \begin{cases}\operatorname{dist}(x, \Gamma(t)) & \text { for } x \in \Omega^{-}(t), \\ -\operatorname{dist}(x, \Gamma(t)) & \text { elsewhere. }\end{cases}
$$

Note that $\tilde{d}=0$ on $\Gamma_{T}$ and $|\nabla \tilde{d}|=1$ in a neighborhood of $\Gamma_{T}$.

2. Outer expansion: It is reasonable to assume that outside a neighbourhood of $\Gamma_{T}$, $u^{\varepsilon}$ has the expansion

$$
u^{\varepsilon}(x, t)= \pm 1+\varepsilon u_{1}^{ \pm}(x, t)+\varepsilon^{2} u_{2}^{ \pm}(x, t)+\ldots
$$


3. Inner expansion: Near $\Gamma_{T}$, we assume that $u^{\varepsilon}$ has form

$$
u^{\varepsilon}(x, t)=U_{0}(x, t, \xi)+\varepsilon U_{1}(x, t, \xi)+\varepsilon^{2} U_{2}(x, t, \xi)+\ldots
$$

Here $U_{j}(x, t, z), j \geq 0$ are defined for $x \in \Omega, t \geq 0, z \in \mathbb{R}$ and $\xi:=\tilde{d}(x, t) / \varepsilon$.

4. Normalization conditions: The stretched space variable $\xi$ gives exactly the right spatial scaling to describe the rapid transition between the regions $\left\{u^{\varepsilon} \approx-1\right\}$ and $\left\{u^{\varepsilon} \approx 1\right\}$. We normalize $U_{0}$ in such a way that

$$
U_{0}(x, t, 0)=0 .
$$

5. Matching conditions: For $\xi \rightarrow \pm \infty$, we require two expansions (3.4) and (3.5) to be consistent, i.e.

$$
U_{0}(x, t,-\infty)=1, \quad U_{0}(x, t,+\infty)=-1 ;
$$

and

$$
U_{k}(x, t,-\infty)=u_{k}^{+}(x, t), \quad U_{k}(x, t,+\infty)=u_{k}^{-}(x, t)
$$

for all $k \geq 1$.

Formal interface motion equation We will substitute the inner expansion (3.5) into (1.5b). We will then compare the terms of the same order to determine equations of $U_{0}$ and $U_{1}$. To that purpose, we start by some computations.

$$
\begin{aligned}
u_{t}^{\varepsilon} & =U_{0 t}+U_{0 z} \frac{\widetilde{d_{t}}}{\varepsilon}+\varepsilon U_{1 t}+U_{1 z} \widetilde{d}_{t}+\ldots, \\
\nabla u^{\varepsilon} & =\nabla U_{0}+U_{0 z} \frac{\nabla \widetilde{d}}{\varepsilon}+\varepsilon \nabla U_{1}+U_{1 z} \nabla \widetilde{d}+\ldots, \\
\Delta u^{\varepsilon} & =\Delta U_{0}+2 \frac{\nabla \widetilde{d}}{\varepsilon} \cdot \nabla U_{0 z}+U_{0 z} \frac{\Delta \widetilde{d}}{\varepsilon}+U_{0 z z} \frac{|\nabla \widetilde{d}|}{\varepsilon^{2}}+\varepsilon \Delta U_{1} \\
& +2 \nabla \widetilde{d} \cdot \nabla U_{1 z}+U_{1 z} \Delta \widetilde{d}+U_{1 z z} \frac{|\nabla \widetilde{d}|}{\varepsilon}+\ldots, \\
f\left(u^{\varepsilon}\right) & =f\left(U_{0}\right)+\varepsilon f^{\prime}\left(U_{0}\right) U_{1}+O\left(\varepsilon^{2}\right), \\
\mu^{\varepsilon} & =\mu+O(\varepsilon) .
\end{aligned}
$$

Substituting $u_{t}^{\varepsilon}, \Delta u^{\varepsilon}, f\left(u^{\varepsilon}\right), \mu^{\varepsilon}$ in (1.5b), collecting all terms of order $\varepsilon^{-2}$ then yields

$$
\left\{\begin{array}{l}
U_{0 z z}+f\left(U_{0}\right)=0 \\
U_{0}(-\infty)=1, \quad U_{0}(0)=0, \quad U_{0}(+\infty)=-1 .
\end{array}\right.
$$

This problem has a unique solution $U_{0}$. Furthermore, $U_{0}$ is independent of $(x, t)$, i.e. $U_{0}(x, t, z)=U_{0}(z)$ and thus, we write $U_{0}^{\prime}, U_{0}^{\prime \prime}$ instead of $U_{0 z}, U_{0 z z}$. We have the following lemma. 
Lemma 3.1. The solution $U_{0}$ of equation (3.6) also fulfills the differential equation

$$
U_{0}^{\prime}=-\sqrt{2 W\left(U_{0}\right)}
$$

As a consequence, $\int_{\mathbb{R}}\left(U_{0}^{\prime}(z)\right)^{2} d z$ can be written in the form:

$$
\int_{\mathbb{R}}\left(U_{0}^{\prime}(z)\right)^{2} d z=\sqrt{2} \int_{-1}^{1} \sqrt{W(s)} d s .
$$

Proof. Multiplying the above mentioned differential equation (3.6) for $U_{0}$ by $U_{0}^{\prime}$, we get

$$
U_{0}^{\prime \prime} U_{0}^{\prime}+f\left(U_{0}\right) U_{0}^{\prime}=0 .
$$

Keeping in mind that $W^{\prime}(u)=-f(u),(3.7)$ can be read as

$$
\left(\frac{\left(U_{0}^{\prime}\right)^{2}}{2}\right)^{\prime}-\left(W\left(U_{0}\right)\right)^{\prime}=0
$$

Integrating this equation from $-\infty$ to $z$, we obtain

$$
\frac{\left(U_{0}^{\prime}(z)\right)^{2}}{2}=W\left(U_{0}(z)\right)
$$

Moreover, $U_{0}$ is non increasing, therefore, we deduce that

$$
U_{0}^{\prime}(z)=-\sqrt{2 W\left(U_{0}(z)\right)} .
$$

Consequently, we have

$$
\int_{\mathbb{R}}\left(U_{0}^{\prime}(z)\right)^{2} d z=-\int_{\mathbb{R}} U_{0}^{\prime}(z) \sqrt{2 W\left(U_{0}(z)\right)} d z=\sqrt{2} \int_{-1}^{1} \sqrt{W(s)} d s .
$$

This completes the proof of Lemma 3.1.

We now collect the terms of order $\varepsilon^{-1}$ in the substituted equation (1.5b). Because we have $|\nabla \widetilde{d}|=1$ in a neighbourhood of $\Gamma(t)$, we obtain

$$
U_{1 z z}+f^{\prime}\left(U_{0}\right) U_{1}=U_{0}^{\prime}\left(\alpha \widetilde{d}_{t}-\Delta \widetilde{d}\right)-\mu .
$$

A solvability condition for this equation is given by the following lemma.

Lemma 3.2 (see Lemma 2.2 in Ref. 1). Let $A(z)$ be a bounded function for $z \in \mathbb{R}$. Then the existence of a solution $\phi$ for the problem

$$
\left\{\begin{array}{l}
\phi_{z z}+f^{\prime}\left(U_{0}(z)\right) \phi=A(z), z \in \mathbb{R} \\
\phi(0)=0, \phi \in L^{\infty}(\mathbb{R})
\end{array}\right.
$$

is equivalent to

$$
\int_{\mathbb{R}} A(z) U_{0}^{\prime}(z) d z=0 .
$$

Therefore, the existence of a solution $U_{1}$ of (3.10) is equivalent to

$$
\int_{\mathbb{R}}\left[\left(U_{0}^{\prime}\right)^{2}(z)\left(\alpha \widetilde{d}_{t}-\Delta \widetilde{d}\right)(x, t)-\mu(x, t) U_{0}^{\prime}(z)\right] d z=0
$$


for all $(x, t)$ in a neighbourhood of the interface $\Gamma_{T}$. Thus,

$$
\left(\alpha \widetilde{d}_{t}-\Delta \widetilde{d}\right)(x, t)=\frac{\mu(x, t) \int_{\mathbb{R}} U_{0}^{\prime}(z) d z}{\int_{\mathbb{R}}\left(U_{0}^{\prime}(z)\right)^{2} d z}=-\frac{2 \mu(x, t)}{\int_{\mathbb{R}}\left(U_{0}^{\prime}(z)\right)^{2} d z} .
$$

It follows from Lemma 3.1 that

$$
\left(\alpha \widetilde{d}_{t}-\Delta \widetilde{d}\right)(x, t)=-\frac{\sqrt{2} \mu(x, t)}{\int_{-1}^{1} \sqrt{W(s)} d s} .
$$

Note that, on $\Gamma(t)$ we have $n=\left.n^{+}\right|_{\Gamma}=\nabla \widetilde{d}, \kappa=\frac{\operatorname{div}(n)}{N-1}=\frac{\Delta \widetilde{d}}{N-1}$, and $\tilde{d}_{t}=-V_{n}$. Therefore, we deduce that $\Gamma(t)$ satisfies indeed the interface motion equation (1.7b):

$$
\alpha V_{n}=-(N-1) \kappa+\frac{\sqrt{2} \mu}{\int_{-1}^{1} \sqrt{W(s)} d s}=-(N-1) \kappa+\mathcal{C} \mu \quad \text { on } \Gamma_{T},
$$

where $\mathcal{C}:=\left[\int_{-1}^{1} \sqrt{W(s) / 2} d s\right]^{-1}$.

\subsection{Equations for $\mu, \sigma$}

We will suppose that the following convergence holds in a strong enough sense:

$$
\mu^{\varepsilon} \longrightarrow \mu, \quad \sigma^{\varepsilon} \longrightarrow \sigma
$$

as $\varepsilon \downarrow 0$ and derive the limit of the reaction term in (1.5a) and (1.5c). To that purpose, we first prove a stronger version of Lemma 2.1 by Du et al. in Ref. 22 (see also Refs. 5, 30).

Lemma 3.3. Let $\gamma \subset \subset \Omega$ be a smooth hypersurface without boundary, $d$ be the signed distance to $\gamma$, and let $g \in L^{1}(\mathbb{R})$. Furthermore, let $\phi^{\varepsilon} \in L^{\infty}(\Omega)$ and $V \subset \Omega$ be a neighborhood $\gamma$ such that

$$
\begin{aligned}
& \left\|\phi^{\varepsilon}\right\|_{L^{\infty}(\Omega)} \leq C, \\
& \phi^{\varepsilon} \text { is continuous on } V, \\
& \phi^{\varepsilon} \longrightarrow \phi \quad \text { uniformly in } V .
\end{aligned}
$$

We then have

$$
\lim _{\varepsilon \downarrow 0} \frac{1}{\varepsilon} \int_{U} g(d(x) / \varepsilon) \phi^{\varepsilon}(x) \mathrm{d} x=\int_{-\infty}^{\infty} g(\tau) \mathrm{d} \tau \int_{\gamma} \phi
$$

for a small enough neighborhood $U \subset V$ of $\gamma$.

Proof. For simplicity, we prove this lemma in the three-dimensional case and assume that the hypersurface $\gamma$ has a parametrization $\alpha$. More precisely, we assume that there exists an open set $W$ of $\mathbb{R}^{2}$ such that the mapping $\alpha$ from $\bar{W}$ onto $\gamma$ is smooth and that $\alpha^{-1}$ is continuous from $\gamma$ onto $\bar{W}$. We write the function $\alpha$ as

$$
\alpha\left(z_{1}, z_{2}\right)=\left(\alpha_{1}\left(z_{1}, z_{2}\right), \alpha_{2}\left(z_{1}, z_{2}\right), \alpha_{3}\left(z_{1}, z_{2}\right)\right) \text { for all }\left(z_{1}, z_{2}\right) \in \bar{W} .
$$


For $\delta>0$ small enough, we consider $\eta$ from $\bar{W} \times[-\delta, \delta]$ to $\mathbb{R}^{3}$, which satisfies

$$
\left\{\begin{array}{l}
\eta_{\tau}\left(z_{1}, z_{2}, \tau\right)=\nabla d\left(\eta\left(z_{1}, z_{2}, \tau\right)\right), \\
\eta\left(z_{1}, z_{2}, 0\right)=\alpha\left(z_{1}, z_{2}\right) .
\end{array}\right.
$$

We write

$$
\eta\left(z_{1}, z_{2}, \tau\right)=\left(\eta_{1}\left(z_{1}, z_{2}, \tau\right), \eta_{2}\left(z_{1}, z_{2}, \tau\right), \eta_{3}\left(z_{1}, z_{2}, \tau\right)\right)
$$

with $\eta_{i}: \bar{W} \times[-\delta, \delta] \rightarrow \mathbb{R}$. We define $U:=\eta(\{\bar{W} \times[-\delta, \delta]\})$ and choose $\delta$ small enough so that $U \subset V$. Note that

$$
\frac{d}{d \tau} d\left(\eta\left(z_{1}, z_{2}, \tau\right)\right)=\nabla d\left(\eta\left(z_{1}, z_{2}, \tau\right)\right) \eta_{\tau}\left(z_{1}, z_{2}, \tau\right)=\left|\nabla d\left(\eta\left(z_{1}, z_{2}, \tau\right)\right)\right|^{2}=1,
$$

and that $d\left(\eta\left(z_{1}, z_{2}, 0\right)\right)=d\left(\alpha\left(z_{1}, z_{2}\right)\right)=0$. Thus we conclude that $d\left(\eta\left(z_{1}, z_{2}, \tau\right)\right)=$ $\tau$. We define $J\left(z_{1}, z_{2}, \tau\right)$ as the determinant of the Jacobian matrix of $\eta$ at $\left(z_{1}, z_{2}, \tau\right)$ and perform the change of coordinates $\eta\left(z_{1}, z_{2}, \tau\right)=x$ to obtain

$$
\begin{aligned}
\int_{U} g\left(\frac{d(x)}{\varepsilon}\right) & \phi^{\varepsilon}(x) d x \\
& =\int_{-\delta}^{\delta} d \tau \int_{W} g\left(\frac{d\left(\eta\left(z_{1}, z_{2}, \tau\right)\right)}{\varepsilon}\right) \phi^{\varepsilon}\left(\eta\left(z_{1}, z_{2}, \tau\right)\right)\left|J\left(z_{1}, z_{2}, \tau\right)\right| d z_{1} d z_{2} \\
& =\int_{-\delta}^{\delta} d \tau \int_{W} g\left(\frac{\tau}{\varepsilon}\right) \phi^{\varepsilon}\left(\eta\left(z_{1}, z_{2}, \tau\right)\right)\left|J\left(z_{1}, z_{2}, \tau\right)\right| d z_{1} d z_{2}
\end{aligned}
$$

By applying the change of coordinates $\tau=\varepsilon \widetilde{\mathcal{T}}$, we have

$$
\begin{aligned}
\int_{U} g\left(\frac{d(x)}{\varepsilon}\right) & \phi^{\varepsilon}(x) d x \\
= & \varepsilon \int_{-\frac{\delta}{\varepsilon}}^{\frac{\delta}{\varepsilon}} d \widetilde{\tau} \int_{W} g(\widetilde{\tau}) \phi^{\varepsilon}\left(\eta\left(z_{1}, z_{2}, \varepsilon \widetilde{\tau}\right)\right)\left|J\left(z_{1}, z_{2}, \varepsilon \widetilde{\tau}\right)\right| d z_{1} d z_{2} .
\end{aligned}
$$

Therefore,

$$
\begin{aligned}
A_{\varepsilon}:=\frac{1}{\varepsilon} \int_{U} g\left(\frac{d(x)}{\varepsilon}\right) & \phi^{\varepsilon}(x) d x \\
& =\int_{-\infty}^{\infty} \int_{W} \mathbf{1}_{\left(-\frac{\delta}{\varepsilon}, \frac{\delta}{\varepsilon}\right)}(\widetilde{\tau}) g(\widetilde{\tau}) \phi^{\varepsilon}\left(\eta\left(z_{1}, z_{2}, \varepsilon \widetilde{\tau}\right)\right)\left|J\left(z_{1}, z_{2}, \varepsilon \widetilde{\tau}\right)\right| d \widetilde{\tau} d z_{1} d z_{2} .
\end{aligned}
$$

In the following, we will apply the dominated convergence theorem to deduce the limit of $A_{\varepsilon}$ as $\varepsilon \downarrow 0$. Set

$$
H_{\varepsilon}\left(z_{1}, z_{2}, \widetilde{\tau}\right):=\mathbf{1}_{\left(-\frac{\delta}{\varepsilon}, \frac{\delta}{\varepsilon}\right)}(\widetilde{\tau}) g(\widetilde{\tau}) \phi^{\varepsilon}\left(\eta\left(z_{1}, z_{2}, \varepsilon \widetilde{\tau}\right)\right)\left|J\left(z_{1}, z_{2}, \varepsilon \widetilde{\tau}\right)\right| .
$$

For $-\frac{\delta}{\varepsilon} \leq \widetilde{\tau} \leq \frac{\delta}{\varepsilon}$, we have $-\delta \leq \varepsilon \widetilde{\tau} \leq \delta$, so that for all $\varepsilon>0$

$$
\left|\mathbf{1}_{\left(-\frac{\delta}{\varepsilon}, \frac{\delta}{\varepsilon}\right)}(\widetilde{\tau})\right| J\left(z_{1}, z_{2}, \varepsilon \widetilde{\tau}\right)|| \leq \sup _{z_{1}, z_{2} \in \bar{W},-\delta \leq \tau \leq \delta}\left|J\left(z_{1}, z_{2}, \tau\right)\right|=: C_{1} .
$$


Moreover, $\left\|\phi^{\varepsilon}\right\|_{L^{\infty}(\Omega)} \leq C$ for all $\varepsilon>0$, therefore,

$$
\left|H_{\varepsilon}\left(z_{1}, z_{2}, \widetilde{\tau}\right)\right| \leq C|g(\widetilde{\tau})| \quad \text { on } \bar{W} \times \mathbb{R} .
$$

Next, since $\phi^{\varepsilon}$ converges uniformly to $\phi$ on $U$ and since $J$ is continuous, we have for all $\widetilde{\tau} \in \mathbb{R},\left(z_{1}, z_{2}\right) \in W$,

$$
\begin{gathered}
\mathbf{1}_{\left(-\frac{\delta}{\varepsilon}, \frac{\delta}{\varepsilon}\right)}(\widetilde{\tau}) \phi^{\varepsilon}\left(\eta\left(z_{1}, z_{2}, \varepsilon \widetilde{\tau}\right)\right) \rightarrow \phi\left(\eta\left(z_{1}, z_{2}, 0\right)\right)=\phi\left(\alpha\left(z_{1}, z_{2}\right)\right), \\
J\left(z_{1}, z_{2}, \varepsilon \widetilde{\tau}\right) \rightarrow J\left(z_{1}, z_{2}, 0\right),
\end{gathered}
$$

as $\varepsilon \downarrow 0$. It follows that as $\varepsilon \downarrow 0$,

$$
H_{\varepsilon}\left(z_{1}, z_{2}, \widetilde{\tau}\right) \rightarrow g(\widetilde{\tau}) \phi\left(\eta\left(z_{1}, z_{2}, 0\right)\right)\left|J\left(z_{1}, z_{2}, 0\right)\right| \text { for all } \widetilde{\tau} \in \mathbb{R},\left(z_{1}, z_{2}\right) \in W .
$$

Combining (3.16) and (3.17), we have

$$
\lim _{\varepsilon \downarrow 0} A_{\varepsilon}=\int_{-\infty}^{\infty} g(\widetilde{\tau}) d \widetilde{\tau} \int_{W} \phi\left(\alpha\left(z_{1}, z_{2}\right)\right)\left|J\left(z_{1}, z_{2}, 0\right)\right| d z_{1} d z_{2} .
$$

Next, we computes $\left|J\left(z_{1}, z_{2}, 0\right)\right|$. For this purpose, we write

$$
\begin{aligned}
\frac{\partial \eta}{\partial z_{1}} & =\left(\frac{\partial \eta_{1}}{\partial z_{1}}, \frac{\partial \eta_{2}}{\partial z_{1}}, \frac{\partial \eta_{3}}{\partial z_{1}}\right), \\
\frac{\partial \eta}{\partial z_{2}} & =\left(\frac{\partial \eta_{1}}{\partial z_{2}}, \frac{\partial \eta_{2}}{\partial z_{2}}, \frac{\partial \eta_{3}}{\partial z_{2}}\right), \\
\frac{\partial \eta}{\partial \tau} & =\left(\frac{\partial \eta_{1}}{\partial \tau}, \frac{\partial \eta_{2}}{\partial \tau}, \frac{\partial \eta_{3}}{\partial \tau}\right) .
\end{aligned}
$$

Note that $\frac{\partial \eta}{\partial \tau}\left(z_{1}, z_{2}, 0\right)$ is the outer normal vector to $\gamma$ at the point $\eta\left(z_{1}, z_{2}, 0\right)=$ $\alpha\left(z_{1}, z_{2}\right)$ and that $\left\{\frac{\partial \eta}{\partial u}\left(z_{1}, z_{2}, 0\right), \frac{\partial \eta}{\partial v}\left(z_{1}, z_{2}, 0\right)\right\}$ is a basis of the tangent space of $\gamma$ at point $\eta\left(z_{1}, z_{2}, 0\right)=\alpha\left(z_{1}, z_{2}\right)$. Therefore,

$$
\begin{aligned}
\left|J\left(z_{1}, z_{2}, 0\right)\right| & =\left|\left(\frac{\partial \eta}{\partial z_{1}} \wedge \frac{\partial \eta}{\partial z_{2}}\right) \cdot \frac{\partial \eta}{\partial \tau}\right|=\left|\frac{\partial \eta}{\partial z_{1}} \wedge \frac{\partial \eta}{\partial z_{2}}\right|\left|\frac{\partial \eta}{\partial \tau}\right| \\
& =\left|\frac{\partial \eta}{\partial z_{1}} \wedge \frac{\partial \eta}{\partial z_{2}}\right|\left|\nabla d\left(\eta\left(z_{1}, z_{2}, 0\right)\right)\right|=\left|\frac{\partial \eta}{\partial z_{1}} \wedge \frac{\partial \eta}{\partial z_{2}}\right|\left(z_{1}, z_{2}, 0\right) \\
& =\left|\frac{\partial \alpha}{\partial z_{1}} \wedge \frac{\partial \alpha}{\partial z_{2}}\right|\left(z_{1}, z_{2}\right)
\end{aligned}
$$

where $\wedge$ is the vector product. This together with (3.18) implies that

$$
\lim _{\varepsilon \downarrow 0} A_{\varepsilon}=\int_{-\infty}^{\infty} g(\widetilde{\tau}) d \widetilde{\tau} \int_{W} \phi\left(\alpha\left(z_{1}, z_{2}\right)\right)\left|\frac{\partial \alpha}{\partial z_{1}} \wedge \frac{\partial \alpha}{\partial z_{2}}\right| d z_{1} d z_{2} .
$$

On the other hand, in view of the definition of the integral of surface (see Eq. (131), p. 283 in Ref. 36), we have

$$
\int_{\gamma} \phi d \gamma=\int_{W} \phi\left(\alpha\left(z_{1}, z_{2}\right)\right)\left|\frac{\partial \alpha}{\partial z_{1}} \wedge \frac{\partial \alpha}{\partial z_{2}}\right| d z_{1} d z_{2}
$$


Therefore,

$$
\lim _{\varepsilon \downarrow 0} A_{\varepsilon}=\int_{-\infty}^{\infty} g(\widetilde{\tau}) d \widetilde{\tau} \int_{\gamma} \phi
$$

which completes the proof of the lemma.

Application to reaction term: Now we apply Lemma 3.3 to formally compute the limit as $\varepsilon \downarrow 0$ of

$$
\frac{1}{\varepsilon} \int_{0}^{T} \int_{\Omega} p\left(u^{\varepsilon}\right)\left(\sigma^{\varepsilon}-\delta \mu^{\varepsilon}\right) \psi, \quad \text { for } \psi \in \mathcal{F}_{T} .
$$

Because of the outer and inner expression of $u^{\varepsilon}$ in (3.4) and (3.5), we deduce that for $\varepsilon$ small enough

$$
u^{\varepsilon}(x, t) \approx \begin{cases} \pm 1 & \text { if }(x, t) \text { is far from } \Gamma_{T} \\ U_{0}\left(\frac{\tilde{d}(x, t)}{\varepsilon}\right) & \text { if }(x, t) \text { is closed to } \Gamma_{T} .\end{cases}
$$

Therefore

$$
p\left(u^{\varepsilon}(x, t)\right) \approx \begin{cases}0 & \text { if }(x, t) \text { is far from } \Gamma_{T} \\ p\left(U_{0}\left(\frac{\tilde{d}(x, t)}{\varepsilon}\right)\right) & \text { if }(x, t) \text { is closed to } \Gamma_{T} .\end{cases}
$$

Thus we can apply Lemma 3.3 by setting

$$
g(\xi):=p\left(U_{0}(\xi)\right) \quad \text { and } \quad \phi^{\varepsilon}:=\left(\sigma^{\varepsilon}-\delta \mu^{\varepsilon}\right) \psi,
$$

where $\psi \in \mathcal{F}_{T}$. This yields

$$
\begin{aligned}
P^{0}(\psi(t)) & :=\lim _{\varepsilon \downarrow 0} \frac{1}{\varepsilon} \int_{\Omega} p\left(u^{\varepsilon}\right)\left(\sigma^{\varepsilon}-\delta \mu^{\varepsilon}\right) \psi=\lim _{\varepsilon \downarrow 0} \frac{1}{\varepsilon} \int_{U(t)} p\left(u^{\varepsilon}\right)\left(\sigma^{\varepsilon}-\delta \mu^{\varepsilon}\right) \psi \\
& =\int_{-\infty}^{\infty} p\left(U_{0}(\xi)\right) \mathrm{d} \xi \int_{\Gamma(t)}(\sigma(t)-\delta \mu(t)) \psi(t),
\end{aligned}
$$

where $U(t)$ is a small enough neighborhood of $\Gamma(t)$. Recalling that in view of the definition of $p$ and of Lemma 3.1

$$
p\left(U_{0}\right)=2 p_{0} \sqrt{W\left(U_{0}\right)}=-\sqrt{2} p_{0} U_{0}^{\prime}
$$

we get

$$
\begin{aligned}
P^{0}(\psi(t)) & =-\sqrt{2} p_{0} \int_{-\infty}^{\infty} U_{0}^{\prime}(\xi) \mathrm{d} \xi \int_{\Gamma(t)}(\sigma(t)-\delta \mu(t)) \psi(t) \\
& =2 \sqrt{2} p_{0} \int_{\Gamma(t)}(\sigma(t)-\delta \mu(t)) \psi(t) .
\end{aligned}
$$


Hence, we formally conclude that, for all $\psi \in \mathcal{F}_{T}$

$$
\lim _{\varepsilon \downarrow 0} \frac{1}{\varepsilon} \int_{0}^{T} \int_{\Omega} p\left(u^{\varepsilon}\right)\left(\sigma^{\varepsilon}-\delta \mu^{\varepsilon}\right) \psi=2 \sqrt{2} p_{0} \int_{0}^{T} \mathrm{~d} t \int_{\Gamma(t)}(\sigma(t)-\delta \mu(t)) \psi(t) .
$$

Conclusion: Now, we recall the definition of a weak solution of the equation for $\mu^{\varepsilon}:$

$$
\begin{aligned}
\int_{0}^{T} \int_{\Omega}\left(-\alpha \mu^{\varepsilon}-u^{\varepsilon}\right) \psi_{t}-\int_{\Omega}\left(\alpha \mu_{0}^{\varepsilon}+\right. & \left.u_{0}^{\varepsilon}\right) \psi(0) \\
& =\int_{0}^{T} \int_{\Omega}\left(\mu^{\varepsilon} \Delta \psi+\varepsilon^{-1} p\left(u^{\varepsilon}\right)\left(\sigma^{\varepsilon}-\delta \mu^{\varepsilon}\right) \psi\right)
\end{aligned}
$$

for $\psi$ in $\mathcal{F}_{T}$ and take the limit $\varepsilon \rightarrow 0$ on both sides, to obtain in view of (3.19)

$$
\begin{aligned}
& \int_{0}^{T} \int_{\Omega}(-\alpha \mu-u) \psi_{t}-\int_{\Omega}\left(\alpha \mu_{0}+u_{0}\right) \psi(0) \\
&=\int_{0}^{T} \int_{\Omega} \mu \Delta \psi+2 \sqrt{2} p_{0} \int_{0}^{T} \mathrm{~d} t \int_{\Gamma(t)}(\sigma-\delta \mu)(t) \psi(t) .
\end{aligned}
$$

This together a similar argument for the equation for $\sigma$ completes the proof of Theorem 1.4.

\section{Numerical experiments}

In this section we briefly explore the main theoretical result (Theorem 1.4) with 2-D and 3-D numerical experiments. In these experiments we compare numerical simulations obtained for the diffuse-interface model for various $\varepsilon>0$ with the sharpinterface limit evolution. For simplicity, we shall restrict ourselves to cases for which we can obtain the exact solution for the sharp-interface model. This spares us from having to discretize the sharp-interface model.

We restrict ourselves to the parameter values $\alpha=\delta=0$, but include an additional parameter $\beta \geq 0$ to control the inertia of the nutrient. We thus consider:

$$
\begin{aligned}
u_{t} & =\Delta \mu+\varepsilon^{-1} p(u) \sigma, \\
\mu & =-\varepsilon^{-1} f(u)-\varepsilon \Delta u, \\
\beta \sigma_{t} & =\Delta \sigma-\varepsilon^{-1} p(u) \sigma .
\end{aligned}
$$

In particular, we focus on the two limiting cases for $\beta$, because they allow for an exact solution of the corresponding sharp-interface limit. For $\beta=0$, the nutrient evolution is said to be quasi-static, while for $\beta \rightarrow \infty$ the nutrient is simply constant and equal to its initial condition. 


\subsection{Discretization of the diffuse-interface model}

Because (4.1) is a stiff system for small $\varepsilon$, one has to be careful in choosing a time discretization: naive schemes have severe stability requirements. We therefore adopt the recently proposed second-order time-accurate convex-splitting scheme, see Ref. 40, which for (4.1) is given by:

$$
\begin{aligned}
\frac{u^{k+1}-u^{k}}{\tau} & =\Delta \tilde{\mu}+\varepsilon^{-1} \tilde{p}^{k+1 / 2} \frac{\sigma^{k+1}+\sigma^{k}}{2}, \\
\tilde{\mu} & =-\varepsilon^{-1} \tilde{f}^{k+1 / 2}-\varepsilon \Delta \frac{u^{k+1}+u^{k}}{2}-\alpha_{1} \tau \Delta\left(u^{k+1}-u^{k}\right)+\alpha_{2} \tau\left(u^{k+1}-u^{k}\right), \\
\beta \frac{\sigma^{k+1}-\sigma^{k}}{\tau} & =\Delta \frac{\sigma^{k+1}+\sigma^{k}}{2}-\varepsilon^{-1} \tilde{p}^{k+1 / 2} \frac{\sigma^{k+1}+\sigma^{k}}{2},
\end{aligned}
$$

for $k=0,1,2, \ldots$, and where $\tau$ is the time-step size (assumed constant).

This scheme is essentially a Crank-Nicolson scheme with special treatment of the nonlinear terms, in particular, the free energy $W(u)$ is split into a convex $W_{c}(u)$ and concave part $-W_{e}(u)$ thereby inducing a splitting of $f(u)=f_{c}(u)-f_{e}(u)$, which are treated in a more implicit and explicit manner, respectively:

$$
\tilde{f}^{k+1 / 2}:=f_{c}\left(u^{k+1}\right)-\frac{u^{k+1}-u^{k}}{2} f_{c}^{\prime}\left(u^{k+1}\right)-f_{e}\left(u^{k}\right)-\frac{u^{k+1}-u^{k}}{2} f_{e}^{\prime}\left(u^{k}\right) .
$$

The nonlinearity coming from $p(u)$ is treated by extrapolation:

$$
\tilde{p}^{k+1 / 2}:=p\left(\frac{3}{2} u^{k}-\frac{1}{2} u^{k-1}\right),
$$

where for the first time step $(k=0)$, we take $u^{-1}=u^{0}$.

The parameters $\alpha_{1}$ and $\alpha_{2}$ are stabilization parameters. For the system with $\delta>$ 0 and $\beta=1$, it is proven in Ref. 40 that for $\alpha_{1}$ and $\alpha_{2}$ sufficiently large, the above scheme is stable in the sense that the Lyapunov functional $E_{\varepsilon}$ decreases along timediscrete solution orbits. The case $\delta=0$ is open, but numerical results seem to indicate the the scheme's stability is independent of $\delta$. We refer to Ref. 40 for more details on and properties of the scheme.

For the discretization in space, we employ centred finite differences with gridsize $h$, although one may also employ a spectral method or Galerkin finite element method; see for the latter, e.g., Ref. 28.

\subsection{Test case I: Growing circular tumor}

For the first test case, we consider a growing circular tumor, which start at radius $\rho_{0}$, and a quasi-static nutrient evolution $(\beta=0)$.

\subsubsection{Exact solution of sharp-interface limit}

Before going into the comparison, let us first describe the exact solution of the sharpinterface limit. When it comes to the sharp-interface model, the initial condition is 
given by (in polar coordinates):

$$
\Gamma(0)=\Gamma_{0}=\left\{r=\rho_{0}\right\},
$$

or in terms of the radius $\rho(t)$ of the tumor:

$$
\rho(0)=\rho_{0} \text {. }
$$

For $\beta=0$, one may verify that the sharp-interface limit in Theorem 1.2 simplifies to (note that $\mu$ reduces to a constant in space for constant $\kappa$ ):

$$
\begin{aligned}
\Delta \sigma & =0 & & \text { in } Q_{T}^{+} \cup Q_{T}^{-}, \\
\llbracket \sigma \rrbracket & =0 & & \text { on } \Gamma_{T}, \\
V_{n} & =-\sqrt{2} p_{0} \sigma & & \text { on } \Gamma_{T}, \\
\llbracket \frac{\partial \sigma}{\partial n} \rrbracket & =-2 \sqrt{2} p_{0} \sigma & & \text { on } \Gamma_{T},
\end{aligned}
$$

To close the above system we need to specify a suitable boundary condition for $\sigma$. We assume that the boundary condition is a (time-dependent) axi-symmetric Dirichlet condition:

$$
\sigma(x, t)=\sigma_{\mathrm{D}}(|x|, t), \quad \text { on } \partial \Omega \times(0, T] .
$$

Because of axi-symmetry, the initial circular tumor will indeed remain circular and its radius evolves according to:

$$
\rho^{\prime}(t)=-V_{n} .
$$

Eqs. (4.2a)-(4.2g) fully specify the sharp-interface model in terms of $\{\rho, \sigma\}$. Its solution can be found in an exact manner for a smart choice of $\sigma_{\mathrm{D}}$. To be precise, we shall describe a manufactured solution which satisfies all equations except (4.2f), but which then of course specifies $\sigma_{\mathrm{D}}$. The manufactured solution for $\sigma$ consists of two parts (automatically satisfying $(4.2 \mathrm{~b})$ and $(4.2 \mathrm{c})$ ):

$$
\sigma(r, t)= \begin{cases}\sigma_{\rho}(t) & r \leq \rho(t), \\ \sigma_{R}-\frac{\log (r / R)}{\log (\rho(t) / R)}\left(\sigma_{R}-\sigma_{\rho}(t)\right) & r>\rho(t),\end{cases}
$$

where $\sigma_{R}>0$ and $R>\rho_{0}$ are fixed constants. The constant $\sigma_{\rho}(t)$ is fixed by requiring (4.2e) leading to

$$
\sigma_{\rho}(t)=\frac{\sigma_{R}}{1-2 \sqrt{2} p_{0} \rho(t) \log (\rho(t) / R)} .
$$

Finally, $\rho(t)$ is determined by solving (numerically) the ODE that is obtained by combining $(4.2 \mathrm{~g})$ and $(4.2 \mathrm{~d})$, with the initial condition $(4.2 \mathrm{a})$.

Figure 2 shows the evolution of $\rho(t)$ for various settings of the parameters $\sigma_{R}$ and $R$. It can be seen that the growth is more pronounced for larger values of $\sigma_{R}$ and for smaller values of $R$.

Figure 3 shows for one particular set of parameters the evolution of the tumor, as well as the corresponding axi-symmetric distribution of $\sigma$. Notice that at each 

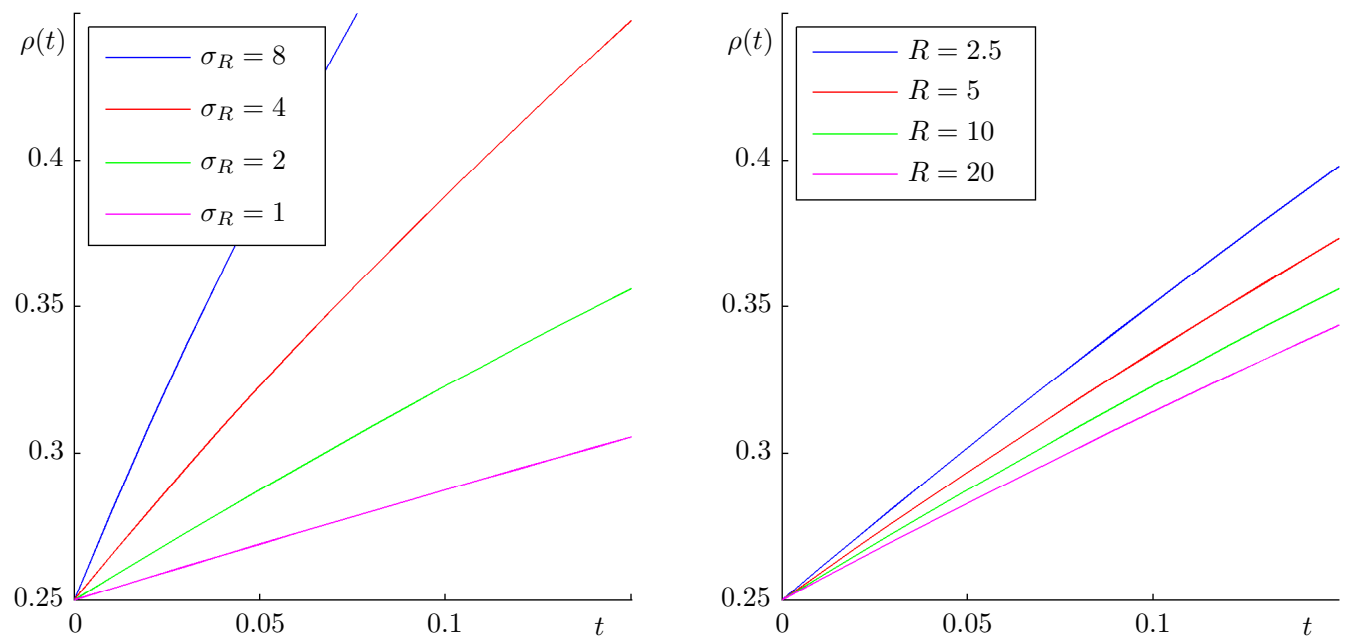

Fig. 2. Test case I: Sharp-interface limit. The radius $\rho(t)$ of a circular tumor which starts at radius $\rho_{0}=1 / 4$, and grows under the influence of a quasi-static nutrient evolution. Three parameters influence the growth: The left graph shows the evolution for parameter value $R=10$ and various $\sigma_{R}$, and the right graph for the parameter value $\sigma_{R}=2$ and various $R$. The proliferation parameter is fixed throughout: $p_{0}=1$.
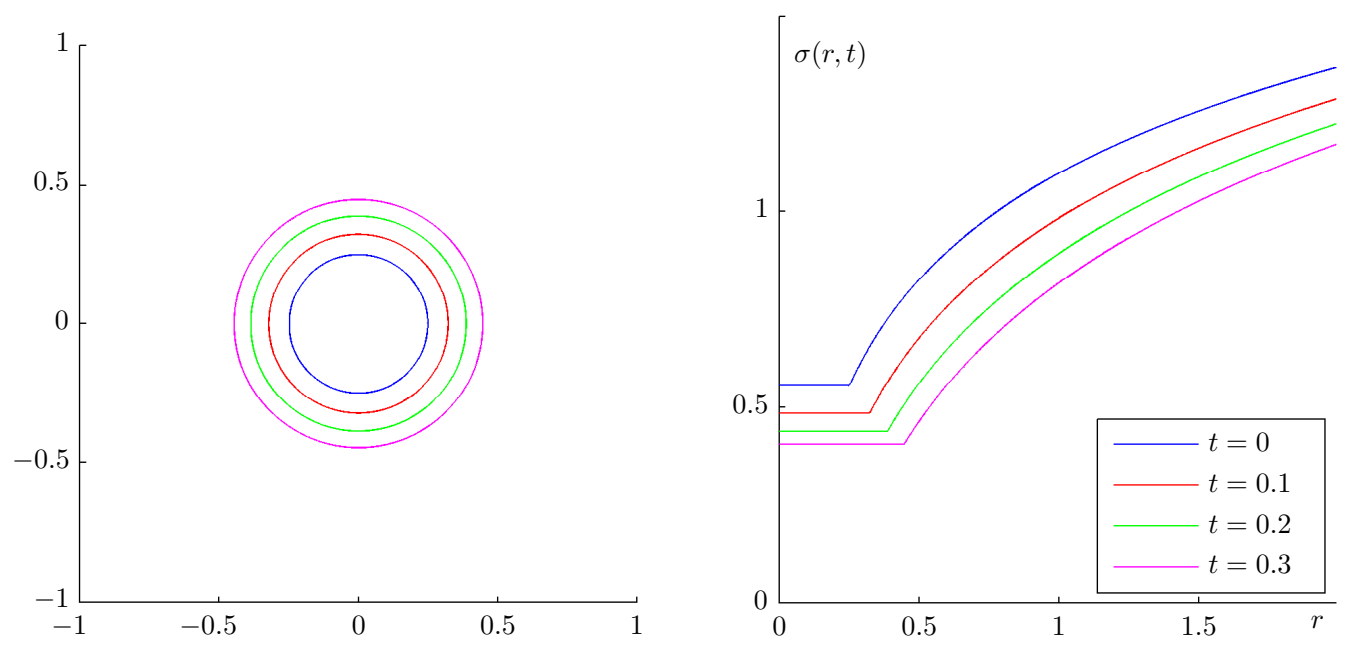

Fig. 3. Test case I: Sharp-interface limit. The left graph shows the tumor at various time instances for $\rho_{0}=1 / 4, p_{0}=1, R=10$, and $\sigma_{R}=2$. The right graph shows the corresponding axi-symmetric nutrient distribution $\sigma(r, t)$.

time $\sigma$ contains two parts: a constant part within the tumor, and a nonconstant part exterior to the tumor. 
Table 1. Test case I: Diffuse-interface model. Settings in the numerical simulations.

\begin{tabular}{lll}
\hline Model & IC / BC & Discretization \\
\hline$\varepsilon=0.15,0.1,0.05$ & $\rho_{0}=1 / 4$ & $\Omega=(-1,1) \times(-1,1)$ \\
$W(u)=\frac{1}{4}\left(1-u^{2}\right)^{2}$ & $\sigma_{R}=2$ & $T=0.075$ \\
$f(u)=-W^{\prime}(u)$ & $R=10$ & $\tau=0.001$ \\
$p_{0}=1$ & & $h=0.003125$ \\
$p(u):$ see Eq. (1.4) & & $\alpha_{1}=\alpha_{2}=2$ \\
$\beta=0$ & & \\
\hline
\end{tabular}
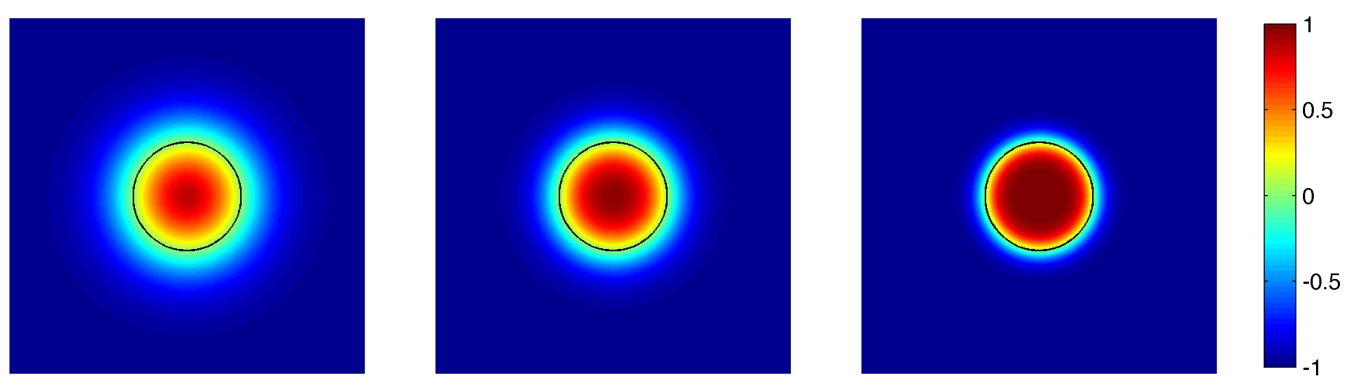

Fig. 4. Test case I: Diffuse-interface model solutions $u$ at $t=0.075$ for $\varepsilon=0.15,0.1$, and 0.05 . The black circle indicate the sharp-interface solution at $t=0.075$.

\subsubsection{Comparison with diffuse-interface model}

We now compare the above exact solution with simulations of the diffuse-interface model. System (4.1) (with $\beta=0$ ) is considered on a square domain subject to natural boundary conditions on $\partial \Omega$ for $u$ and $\mu$, and the Dirichlet boundary condition in (4.2f) for $\sigma$. The initial condition for $u$ is given by:

$$
u(r, 0)=-\tanh \left(\frac{r-\rho_{0}}{\varepsilon \sqrt{2}}\right) .
$$

Details of the choices of all functions and parameters in the numerical simulation can be found in Table $1 .^{\text {a }}$

In Figure 4, we show a comparison of the sharp- and diffuse-interface solutions at the final time, $T=0.075$, for various $\varepsilon$. The colors in these plots display the value for $u$, while the black circle shows the sharp-interface model. It is clear from the plots that for smaller $\varepsilon$ the layers get thinner and converge to the black circle.

The convergence of the diffuse-interface model can be seen more clearly in Figure 4.2.2, which shows the (numerical) radius of the diffuse-interface model versus

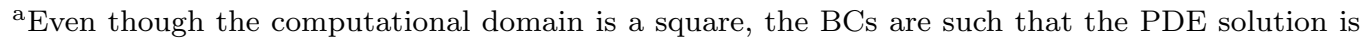
axi-symmetric. Numerical approximations, however, are not exactly axi-symmetric, but we have ensured that they are sufficiently accurate. For example, to compute the radius, we determined the zero level set of the discrete phase field, and computed the average distance of the zero level set to the origin. With the employed mesh, the deviation of the average was less than 0.001. 


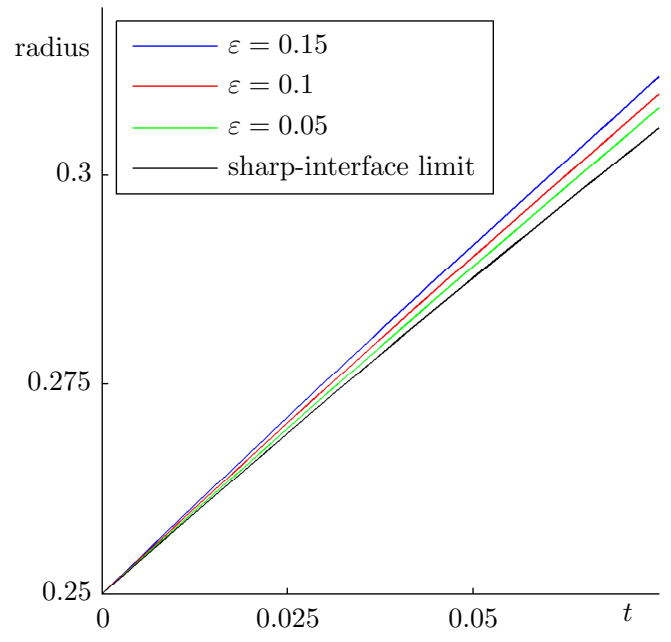

Fig. 5. Test case I: Convergence of the radius of the diffuse-interface model to its identified limit.

time, for various $\varepsilon$. From this figure it can be concluded that the diffuse-interface model converges indeed to our identified limit. While the comparison is, in principle, valid for all times (since no singularity occurs at any time), it should be remarked that for small $\varepsilon$ the simulations are quite sensitive to discretization errors. Such errors may grow at an exponential rate in time (see, e.g., Refs. 23, 38 for a discussion on this), which means that, in practice, comparisons can be made only at (relatively) short time intervals.

\subsection{Test case II: Merging tumors}

In the second test case we consider two separately growing circular (in 2-D) and spherical (in 3-D) tumors, that eventually merge into one. We assume $\beta \rightarrow \infty$, so that $\sigma=\sigma_{0}=$ constant throughout the evolution.

Since $\sigma$ is constant, the sharp-interface model for spherical tumors now reduces essentially to (4.2d):

$$
V_{n}=-\sqrt{2} p_{0} \sigma_{0}
$$

This is known simply as front propagation, which propagates the tumor boundary at a constant velocity.

Let us remark that the presented theory on the sharp-interface limit holds only for smoothly evolving interfaces, and, as such, does not include topological changes. Nevertheless, the sharp interface model of front propagation does allow for an extension beyond topological changes (using viscosity solutions); see, e.g., Ref. 37. We have simply taken the velocity at kinks (when two interfaces touch) as the average of the velocities at both sides. In this manner, it is possible to compare the sharpand diffuse-interface solution beyond topological changes. The aim of this test case 
Table 2. Test case II: Diffuse-interface model. Settings in the numerical simulations in 2-D and 3-D ( $N=2$ and $N=3$, respectively).

\begin{tabular}{lll}
\hline Model & IC / BC & Discretization \\
\hline$\varepsilon=0.2,0.1,0.05(N=2), \varepsilon=0.1(N=3)$ & $\sigma_{0}=2$ & $\Omega=(-1,1)^{N}$ \\
$W(u)=\frac{1}{4}\left(1-u^{2}\right)^{2}$ & $R_{1}=1 / 6$ & $T=0.075$ \\
$f(u)=-W^{\prime}(u)$ & $R_{2}=1 / 4$ & $\tau=0.00025$ \\
$p_{0}=1$ & $d=1 / 5$ & $h=0.025(N=2)$ \\
$p(u):$ see Eq. (1.4) & & $h=0.05(N=3)$ \\
$\beta \rightarrow \infty$ & & $\alpha_{1}=\alpha_{2}=2$ \\
\hline
\end{tabular}

is to demonstrate that both evolutions are very similar, even beyond singularities, which are not covered by the presented theory.

We assume that the two tumors initially have a radius $R_{1}$ and $R_{2}$, and have a gap $d$ between them. The initial condition for $u$ in the diffuse-interface model in 2-D is set as:

$$
\begin{aligned}
u_{0}\left(x_{1}, x_{2}\right)= & 1-\tanh \frac{\sqrt{\left(x_{1}-\frac{1}{2} \sqrt{2}\left(R_{1}+\frac{d}{2}\right)\right)^{2}+\left(x_{2}+\frac{1}{2} \sqrt{2}\left(R_{1}+\frac{d}{2}\right)\right)^{2}}-R_{1}}{\varepsilon \sqrt{2}} \\
& -\tanh \frac{\sqrt{\left(x_{1}+\frac{1}{2} \sqrt{2}\left(R_{2}+\frac{d}{2}\right)\right)^{2}+\left(x_{2}-\frac{1}{2} \sqrt{2}\left(R_{2}+\frac{d}{2}\right)\right)^{2}}-R_{2}}{\varepsilon \sqrt{2}},
\end{aligned}
$$

while in $3-\mathrm{D}$ it is set as:

$$
\begin{aligned}
& u_{0}\left(x_{1}, x_{2}, x_{3}\right)= \\
& 1-\tanh \frac{\sqrt{\left(x_{1}-\frac{1}{2} \sqrt{2}\left(R_{1}+\frac{d}{2}\right)\right)^{2}+\left(x_{2}+\frac{1}{2} \sqrt{2}\left(R_{1}+\frac{d}{2}\right)\right)^{2}+x_{3}^{2}}-R_{1}}{\varepsilon \sqrt{2}} \\
&-\tanh \frac{\sqrt{\left(x_{1}+\frac{1}{2} \sqrt{2}\left(R_{2}+\frac{d}{2}\right)\right)^{2}+\left(x_{2}-\frac{1}{2} \sqrt{2}\left(R_{2}+\frac{d}{2}\right)\right)^{2}+x_{3}^{2}}-R_{2}}{\varepsilon \sqrt{2}} .
\end{aligned}
$$

The numerical settings are given in Table 2 .

Figure 6 displays the solutions obtained with the diffuse-interface model, for various $\varepsilon$, and by front propagation (see (4.3)). From this figure, one can see that the diffuse-interface model seems to converge towards its identified limit, even beyond the topological change.

Figure 7 shows a 3 -D diffuse-interface simulation which is the 3 -D analogue of the previous 2-D simulation at $\varepsilon=0.1$ (middle row in Figure 6). The 3 -D evolution by front-propagation is also visible in Figure 7 (bottom row). In general, compared to the sharp-interface model, one may observe a blending/smoothing of the two spheres in the diffuse-interface model which seems to decrease with $\varepsilon$. 

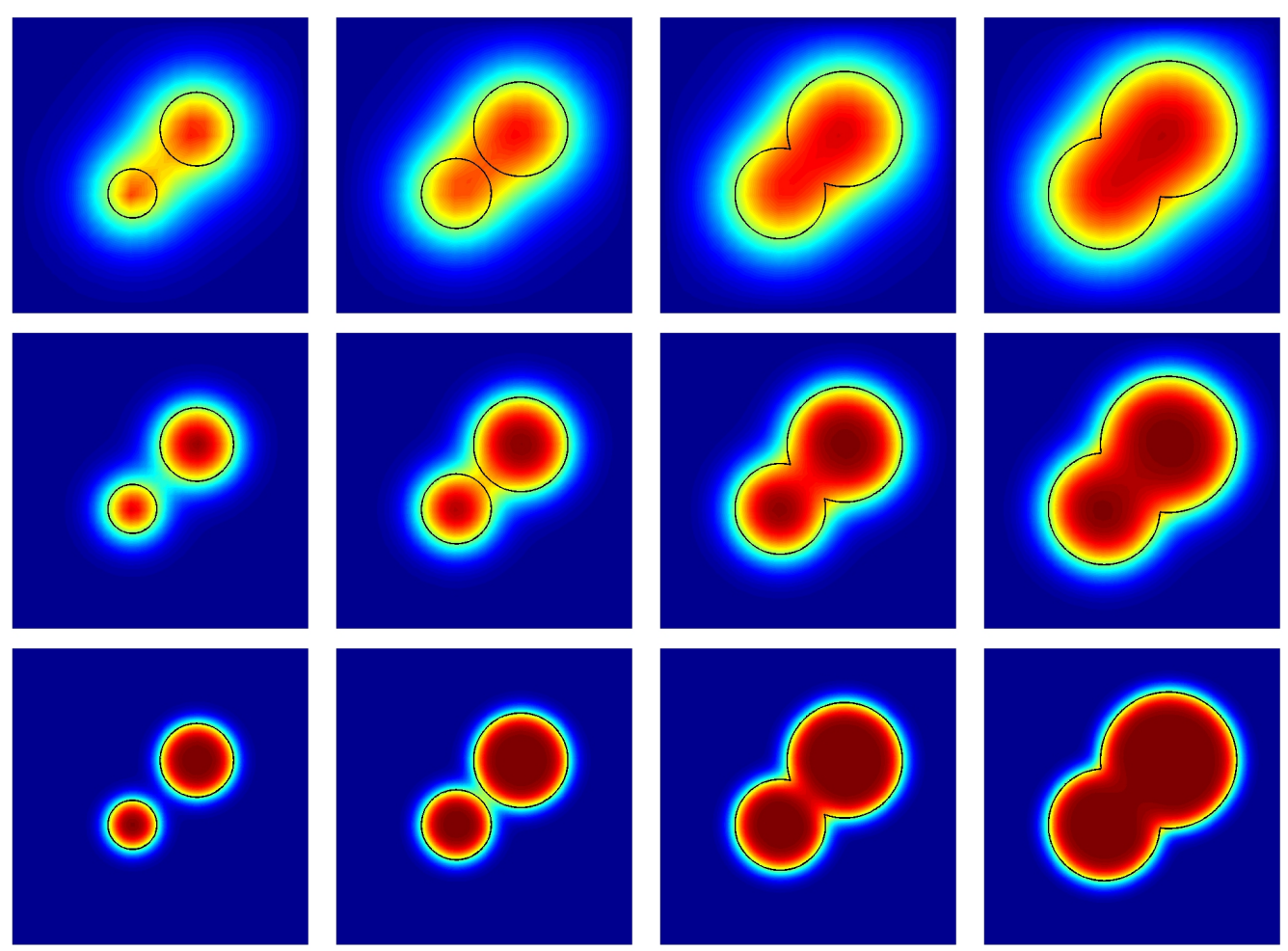

Fig. 6. Test case II: Two merging tumors in 2-D simulated with the diffuse-interface model with $\varepsilon=0.2$ (top row), $\varepsilon=0.1$ (middle row), and $\varepsilon=0.05$ (bottom row). The columns represent subsequent time instances $t=0,0.025,0.05$ and 0.075 . The black curves are obtained from the sharp-interface model described by front-propagation.

\section{Conclusions}

As a conclusion, we have presented a formal asymptotic method for deriving the sharp-interface limit of a diffuse-interface tumor growth model. This limit depends on the precise scaling which we have considered, and we have chosen the scaling corresponding to the case where the mean curvature of the tumor boundary explicitly appears in the limit problem. Moreover, in the limit, the reactive terms of the model collapse to the interface, which is different than in other models where the reactive terms remain as bulk contributions.

Whereas the tumor growth model has the form of a generalized Cahn-Hilliard equation, we have first embedded it into a phase-field model $(\alpha>0)$ for which we found it more intuitive to derive the corresponding limit problem. Setting the parameter $\alpha$ to zero in the corresponding result then yields the limit of the original Cahn-Hilliard type system. Even if it is not vital for results presented in this paper, it is nevertheless interesting to note that the introduction of the phase-field model gives the model a parabolic structure with respect to $\mu$ which can be useful for the analysis of such systems. ${ }^{16}$ 

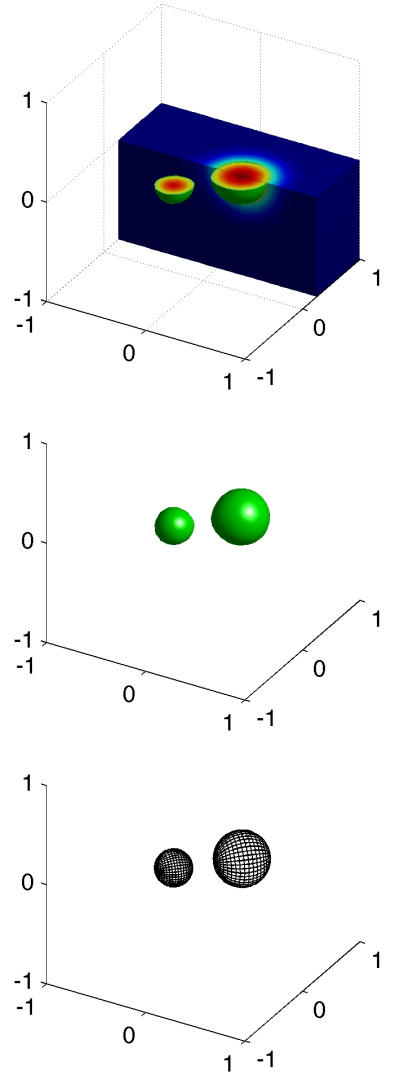
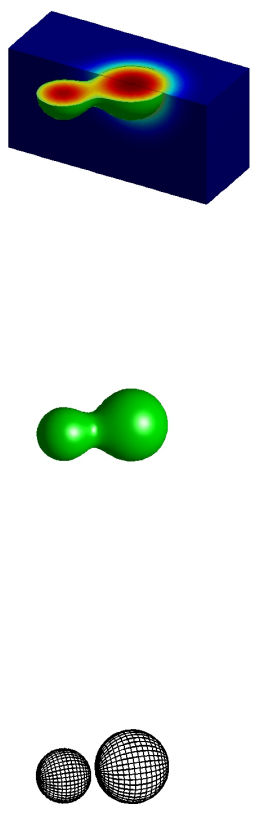
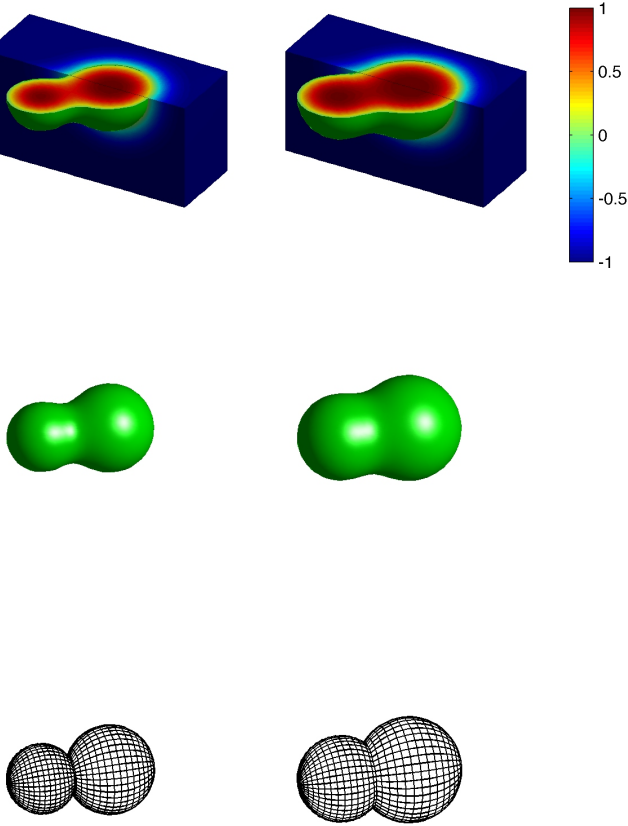

Fig. 7. Test case II: Two merging tumors in 3-D simulated with the diffuse-interface model with $\varepsilon=0.1$. The columns represent subsequent time instances $t=0,0.025,0.05$ and 0.075 . The top row displays a cut-away view of the phase-field $u$, the middle row shows the zero-level set $\{u=0\}$, and the bottom row displays the sharp-interface model described by front-propagation (see (4.3)). This simulation is a three-dimensional analogue of the one depicted in the middle row of Fig. 6.

A validation of the identified limit model was carried out by comparing its solutions in simplified settings with numerical computations of the diffuse interface model for decreasing values of $\varepsilon$. With this work, we have provided a unification of modeling frameworks for tumor growth by connecting mixture-based diffuseinterface models to those with evolving sharp boundaries. We hope that this helps researchers in mathematical oncology in understanding and applying models, and developing better algorithms.

\section{Acknowledgment}

The support of this work by the Netherlands Organisation for Scientific Research (NWO) via the Innovational Research Incentives Scheme (IRIS), Veni grant 639.031.033, is gratefully acknowledged. The last author would like to express his thanks to the hospitality of the Université de Paris-Sud, where this research was 
initiated, as well as to Xunxun Wu for useful discussion on the numerical simulations.

\section{References}

1. M. Alfaro, D. Hilhorst, and H. Matano. The singular limit of the Allen-Cahn equation and the FitzHugh-Nagumo system. J. Differential Equations, 245:505-565, 2008.

2. N. D. Alikakos, P. W. Bates, and XinFu Chen. Convergence of the Cahn-Hilliard equation to the Hele-Shaw model. Arch. Ration. Mech. Anal., 128:165-205, 1994.

3. S. M. Allen and J. W. Cahn. A microscopic theory for antiphase boundary motion and its application to anitphase domain coarsening. Acta Metall., 27:1085-1095, 1979.

4. D. Ambrosi and L. Preziosi. On the closure of mass balance models for tumor growth. Math. Models Methods Appl. Sci., 12(5):737-754, 2002.

5. D. M. Anderson, G. B. McFadden, and A. A. Wheeler. Diffuse-interface methods in fluid mechanics. Annu. Rev. Fluid Mech., 30:139-165, 1998.

6. S. Astanin and L. Preziosi. Multiphase models of tumour growth. In N. Bellomo, M. Chaplain, and E. De Angelis, editors, Selected Topics in Cancer Modeling: Genesis, Evolution, Immune Competition, and Therapy, Modeling and Simulation in Science, Engineering and Technology, chapter 9, pages 223-253. Birkhäuser, 2008.

7. H. Byrne. Modelling avascular tumour growth. In L. Preziosi, editor, Cancer Modelling and Simulation, volume 3 of Mathematical Biology and Medicine Series, chapter 4. Chapman \& Hall/CRC, 2003.

8. H. . Byrne and M. A. J. Chaplain. Growth of nonnecrotic tumors in the presence and absence of inhibitors. Math. Biosci., 130:151-181, 1995.

9. H. Byrne and L. Preziosi. Modelling solid tumour growth using the theory of mixtures. Math. Med. Biol., 20:341-366, 2003.

10. H. M. Byrne, J. R. King, and D. L. S. McElwain. A two-phase model of solid tumour growth. Applied Mathematics Letters, 16:567-573, 2003.

11. G. Caginalp. An analysis of a phase field model of a free boundary. Arch. Ration. Mech. Anal., 92:205-245, 1986.

12. G. Caginalp. Stefan and Hele-Shaw type models as asymptotic limits of the phase-field equations. Phys. Rev. A, 39(11):5887-5896, 1989.

13. G. Caginalp and XinFu Chen. Convergence of the phase field model to its sharp interface limits. European J. Appl. Math., 9:417-445, 1998.

14. G. Caginalp, Xinfu Chen, and C. Eck. Numerical tests of a phase field model with second order accuracy. SIAM J. Appl. Math., 68(6):1518-1534, 2008.

15. G. Caginalp and P. C. Fife. Dynamics of layered interfaces arising from phase boundaries. SIAM J. Appl. Math., 48(3):506-518, 1988.

16. P. Colli, G. Gilardi, and D. Hilhorst. On a Cahn-Hilliard type phase field system related to tumor growth. Technical report, arXiv:1401.5943 [math.AP] (2014) 1-21, 2014. Discrete Contin. Dyn. Syst. to appear (2014).

17. V. Cristini, H. B. Frieboes, X. Li, J. S. Lowengrub, P. Macklin, S. Sanga, S. M. Wise, and X. Zheng. Nonlinear modeling and simulation of tumor growth. In N. Bellomo, M. Chaplain, and E. De Angelis, editors, Selected Topics in Cancer Modeling: Genesis, Evolution, Immune Competition, and Therapy, Modeling and Simulation in Science, Engineering and Technology, chapter 6, pages 113-181. Birkhäuser, 2008.

18. V. Cristini, X. Li, J. S. Lowengrub, and S. M. Wise. Nonlinear simulations of solid tumor growth using a mixture model: invasion and branching. J. Math. Biol., 58:723763, 2009 .

19. V. Cristini and J. S. Lowengrub. Multiscale Modeling of Cancer: An Integrated Ex- 
perimental and Mathematical Modeling Approach. Cambridge University Press, Cambridge, 2010.

20. V. Cristini, J. S. Lowengrub, and Qing Nie. Nonlinear simulation of tumor growth. J. Math. Biol., 46:191-224, 2003.

21. M. C. Delfour and J.-P. Zolésio. Shapes and Geometries: Analysis, Differential Calculus, and Optimization, volume 4 of SIAM Series on Advances in Design and Control. Society for Industrial and Applied Mathematics, 2001.

22. Qiang Du, Chun Liu, R. Ryham, and Xiaoqiang Wang. A phase field formulation of the Willmore problem. Nonlinearity, 18:1249-1267, 2005.

23. K. Eriksson, C. Johnson, and A. Logg. Adaptive computational methods for parabolic problems. In E. Stein, R. de Borst, and T. J. R. Hughes, editors, Encyclopedia of Computational Mechanics, volume 1: Fundamentals, chapter 24, pages 675-702. John Wiley \& Sons, 2004.

24. Xiaobing Feng and A. Prohl. Numerical analysis of the Cahn-Hilliard equation and approximation for the Hele-Shaw problem. Interfaces Free Bound., 7:1-28, 2005.

25. P. C. Fife. Dynamics of Internal Layers and Diffusive Interfaces, volume 53 of $C B M S$ NSF Reginonal Conference Series in Applied Mathematics. Society of Industrial and Applied Mathematics (SIAM), Philadelphia, Pennsylvania, 1988.

26. H. P. Greenspan. On the growth and stability of cell cultures and solid tumors. J. Theoret. Biol., 56:229-242, 1976.

27. A. Hawkins-Daarud, S. Prudhomme, K. G. van der Zee, and J. T. Oden. Bayesian calibration, validation, and uncertainty quantification of diffuse interface models of tumor growth. J. Math. Biol., 67:1457-1485, 2013.

28. A. Hawkins-Daarud, K. G. van der Zee, and J. T. Oden. Numerical simulation of a thermodynamically consistent four-species tumor growth model. Int. J. Numer. Meth. Biomed. Engng., 28:3-24, 2012.

29. E. A. B. F. Lima, J. T. Oden, and R. C. Almeida. A hybrid ten-species phasefield model of tumor growth. Math. Models Methods Appl. Sci., 2014. DOI: 10.1142/S0218202514500304.

30. J. S. Lowengrub and L. Truskinovsky. Quasi-incompressible Cahn-Hilliard fluids and topological transitions. Proc. R. Soc. Lond. Ser. A Math. Phys. Eng. Sci., 454(1978):2617-2654, 1998.

31. M. Mimura. Reaction-diffusion systems arising in biological and chemical systems: Application of singular limit procedures. In P. Colli and J. F. Rodrigues, editors, Mathematical Aspects of Evolving Interfaces, volume 1812 of Lecture Notes in Mathematics, chapter 3, pages 89-121. Springer, Berlin, 2003. Lectures given at the C.I.M.-C.I.M.E. joint Euro-Summer School held in Madeira, Funchal, Portugal, July 3-9, 2000.

32. Y. Nishiura. Far-From-Equilibrium Dynamics, volume 209 of Translations of Mathematical Monographs, Iwanami Series in Modern Mathematics. American Mathematical Society (AMS), 2002.

33. J. T. Oden, A. Hawkins, and S. Prudhomme. General diffuse-interface theories and an approach to predictive tumor growth modeling. Math. Models Methods Appl. Sci., 20(3):477-517, 2010.

34. R. L. Pego. Front migration in the nonlinear Cahn-Hilliard equation. Proc. R. Soc. Lond. Ser. A Math. Phys. Eng. Sci., 422:261-278, 1989.

35. T. Roose, S. J. Chapman, and P. K. Maini. Mathematical models of avascular tumor growth. SIAM Rev., 49(2):179-208, 2007.

36. W. Rudin. Principles of Mathematical Analysis. International Series in Pure and Applied Mathematics. McGraw-Hill, New York, 3rd edition, 1976.

37. J. A. Sethian. Level Set Methods and Fast Marching Methods: Evolving Interfaces 
in Computational Geometry, Fluid Mechanics, Computer Vision, and Materials Science. Cambridge Monographs on Applied and Computational Mathematics. Cambridge University Press, 2nd edition, 1999.

38. K. G. van der Zee, J. T. Oden, S. Prudhomme, and A. Hawkins-Daarud. Goal-oriented error estimation for Cahn-Hilliard models of binary phase transition. Numer. Methods Partial Differential Equations, 27(1):160-196, 2011.

39. S. M. Wise, J. S. Lowengrub, H. B. Frieboes, and V. Cristini. Three-dimensional multispecies nonlinear tumor growth-I Model and numerical method. J. Theoret. Biol., 253:524-543, 2008.

40. X. Wu, G. J. van Zwieten, and K. G. van der Zee. Stabilized second-order convex splitting schemes for Cahn-Hilliard models with application to diffuse-interface tumorgrowth models. Int. J. Numer. Meth. Biomed. Engng., 30:180-203, 2014. 